\title{
Quantifying horizontal and vertical tracer mass fluxes in an idealized valley during daytime
}

\author{
Daniel Leukauf, Alexander Gohm, and Mathias W. Rotach \\ Institute of Atmospheric and Cryospheric Sciences, University of Innsbruck, Innrain 52f, 6020 Innsbruck, Austria \\ Correspondence to: Daniel Leukauf (daniel.leukauf@uibk.ac.at)
}

Received: 23 April 2016 - Published in Atmos. Chem. Phys. Discuss.: 9 June 2016

Revised: 26 August 2016 - Accepted: 21 September 2016 - Published: 21 October 2016

\begin{abstract}
The transport and mixing of pollution during the daytime evolution of a valley boundary layer is studied in an idealized way. The goal is to quantify horizontal and vertical tracer mass fluxes between four different valley volumes: the convective boundary layer, the slope wind layer, the stable core, and the atmosphere above the valley. For this purpose, large eddy simulations (LES) are conducted with the Weather Research and Forecasting (WRF) model for a quasitwo-dimensional valley. The valley geometry consists of two slopes with constant slope angle and is homogeneous in the along-valley direction. The surface sensible heat flux is horizontally homogeneous and prescribed by a sine function. The initial sounding is characterized by an atmosphere at rest and a constant Brunt-Väisälä frequency. Various experiments are conducted for different combinations of surface heating amplitudes and initial stability conditions. A passive tracer is released with an arbitrary but constant rate at the valley floor and resulting tracer mass fluxes are evaluated between the aforementioned volumes.

As a result of the surface heating, a convective boundary layer is established in the lower part of the valley with a stable layer on top - the so-called stable core. The height of the slope wind layer, as well as the wind speed within, decreases with height due to the vertically increasing stability. Hence, the mass flux within the slope wind layer decreases with height as well. Due to mass continuity, this along-slope mass flux convergence leads to a partial redirection of the flow from the slope wind layer towards the valley centre and the formation of a horizontal intrusion above the convective boundary layer. This intrusion is associated with a transport of tracer mass from the slope wind layer towards the valley centre. A strong static stability and/or weak forcing lead to large tracer mass fluxes associated with this phenomenon.
\end{abstract}

The total export of tracer mass out of the valley atmosphere increases with decreasing stability and increasing forcing. The effects of initial stability and forcing can be combined to a single parameter, the breakup parameter $B$. An analytical function is presented that describes the exponential decrease of the percentage of exported tracer mass with increasing $B$. This study is limited by the idealization of the terrain shape, stratification, and forcing, but quantifies transport processes for a large range of forcing amplitudes and atmospheric stability.

\section{Introduction}

It is well known that slope winds provide a mechanism for the vertical exchange of quantities like heat, moisture, and pollutants between a valley atmosphere and the atmosphere aloft (e.g. Reuten et al., 2007; Schmidli and Rotunno, 2010; Lehner and Gohm, 2010; Schmidli, 2013; Rotach et al., 2015). This exchange can be large during fairweather summer days and the export of up to 3 times the valley air mass has been reported (Henne et al., 2004). However, during stably stratified and low-forcing conditions (i.e. high Brunt-Väisälä frequency and weak surface sensible heat flux), the vertical venting is limited and daily pollution limits are frequently exceeded (e.g. Chazette et al., 2005; Suppan et al., 2007; de Franceschi and Zardi, 2009; Silcox et al., 2012; Chemel et al., 2016). Studies have shown that the flow within the slope wind layer may be redirected horizontally leading to a partial recirculation below peak height (e.g. Vergeiner and Dreiseitl, 1987; Reuten et al., 2007; Princevac and Fernando, 2008; Gohm et al., 2009; Lehner and Gohm, 2010). This mechanism is sometimes called a pollution trap 
(Rendón et al., 2014) and may strongly limit the vertical exchange with the atmosphere above the valley. Stable conditions, which lead to such recirculations, are common in Alpine valleys throughout the whole year, especially during fall and winter, giving rise to the question of how effective this exchange mechanism really is. The bulk export of pollutants is particularly relevant for the air quality inside the valley, but may also have an impact further away since polluted air is transported towards adjacent plains by the plainto-mountain circulation (Wagner et al., 2015a; Lang et al., 2015). Therefore, there is a need for quantifying the vertical and horizontal fluxes of pollutants for different atmospheric stability conditions and different forcing strengths, which is the general goal of this study.

In the early work of Vergeiner and Dreiseitl (1987) on slope and valley winds, recirculations within the valley atmosphere are discussed. According to their work, horizontal motions appear at transition zones near elevated temperature inversions where the depth of the slope wind layer and/or the wind speed is reduced. As a result, the along-slope mass flux in the slope wind layer is reduced. Due to mass continuity, a fraction of the slope flow is recirculated below the inversion towards the valley centre. Air from the valley centre is incorporated into the slope wind layer above the inversion as the along-slope mass flux increases again. More recently, Princevac and Fernando (2008) proposed a different mechanism for the formation of this type of flow splitting below the inversion. According to this study, a flow ascending along the slope leads to convergence if the slope flow at higher levels is either slower or directed downslope. Due to mixing within the slope wind layer, air parcels at the top are negatively buoyant with respect to the air in the valley core and either propagate more slowly upwards or even downwards. This leads to a partial redirection of the flow towards the centre of the valley at mid-valley depth, what is know as horizontal intrusion (HI). This HI is a persistent flow feature, located at the transition zone between slope wind layer and stable core. However, turbulence is predominately generated through shear as the HI penetrates into the stable core. Additionally, turbulence is advected from the slope wind layer towards the centre of the valley. Hence, the HI is a part of the mean flow field but has a turbulence component as well. Depending on slope angle, forcing, and stability, this intrusion may play a role in the erosion of the cold pool in the valley and, hence, the breakup of the valley inversion. In general, HIs appear more likely in regions of changing slope angle, surface forcing, or stability (Reuten et al., 2007). In addition to this HI, transport of heat and pollution out of the slope wind layer occurs at all heights due to turbulent detrainment at the top of the slope wind layer.

In nature, rather rapid changes of stability or surface conditions with height are the norm rather than the exception. For example, in an Alpine valley, over an 18-day period in January, elevated inversions were found during $84 \%$ of the time (Emeis et al., 2007). When favourable conditions are met and pollutants are present at the valley bottom, HIs may create banners of polluted air. Such a case has been studied in the Inn Valley using airborne, in situ observations and a backscatter lidar (Harnisch and Gohm, 2009; Gohm et al., 2009; Schnitzhofer et al., 2009). Three distinguishable banners with elevated aerosol concentrations were found that originated from the sunlit slope of the valley and appeared to be formed by an interaction of the slope wind with elevated inversions. An idealized modelling study based on this case supports this conclusion (Lehner and Gohm, 2010). The presence of elevated layers with increased pollution is not only confined to valleys. Lu and Turco $(1994,1995)$ have studied the transport of pollutants in the coastal environment of the Los Angeles Basin and the Santa Ana Mountains using an atmospheric model. The presence of the sea breeze interacting both with the convective boundary layer (CBL) and the slope wind layer led to complex interactions and the authors identified four different processes responsible for pollution layers. One mechanism is again the intrusion of aerosol-rich air from the mountain slope into an inversion layer.

The intrusion of aerosol and other pollutants into the inversion layer has implications for the definition and determination of the boundary layer height over complex terrain. The CBL is usually defined over flat terrain as a neutrally stratified layer bounded by a super-adiabatic surface layer and topped by an inversion (Sullivan et al., 1998). However, other definitions use the distribution of pollution and define the mixed layer as the layer above ground, in which pollutants released at the surface are mixed within about an hour (Seibert et al., 2000). Since pollutants are also transported vertically by slope winds, and horizontally by detrainment from the slope wind layer and by HIs, this definition is ambiguous for a valley. If multiple aerosol layers exist within a valley, the mixed layer height according to this definition may be unclear (De Wekker and Kossmann, 2015).

Once the pollutants are exported out of the valley, they are partly injected vertically into the free atmosphere above the capping inversion (mountain venting, Fast and Zhong, 1998) or horizontally if the boundary layer top is tilted (advective venting, Kossman et al., 1999). This way, the pollutants are incorporated into the plain-to-mountain circulation and are advected towards the adjacent plain (Wagner et al., 2015a). Valleys embedded in a mountain ridge add another layer of complexity to the system since they develop a slope wind system of their own, which interacts with the plain-tomountain flow Lang et al. (2015). However, before pollutants can be incorporated into the complex flow above the mountains, they have to be advected out of the valley atmosphere, a process that is one subject of this study.

Inside a valley, even without an elevated inversion or a cold-air pool present at sunrise and with a homogeneous surface sensible heat flux, at least one HI is likely to develop during the evolution of the valley boundary layer. This is due to the development of a CBL with a capping inversion, which leads to a sharp transition from a neutral to a stable stratifica- 
tion and enables the development of an HI. For this reason a single HI formed in many previous simulations of the valley boundary layer (e.g. Schmidli and Rotunno, 2010; Serafin and Zardi, 2010; Schmidli, 2013; Rendón et al., 2014; Wagner et al., 2014, 2015a, b; Leukauf et al., 2015).

However, tracer mass fluxes associated with HIs have never been quantified. In this study, we aim to quantifying the horizontal and vertical fluxes of pollutants between CBL, slope wind layer, stable core, and the atmosphere above an idealized valley as well as the total export of tracer mass. This is done for a broad range of atmospheric stability and amplitudes of surface sensible heat flux. The initial atmospheric stability ranges from about one-half to 2 times the stability of the standard atmosphere, and the reference forcing amplitude of $125 \mathrm{~W} \mathrm{~m}^{-2}$ corresponds approximately to the average amplitude of fluxes as observed during a "radiation day" in the Riviera Valley in late August (Rotach et al., 2007). The chosen quasi-two-dimensional valley geometry is a simplified version of the one used by Schmidli (2013). This reference forcing amplitude has been multiplied by the factors $0.5,2$ and 3. Studying both the impact of the initial stratification and forcing amplitude complements the studies of Wagner et al. (2014, 2015a, b), which are concerned with the impact of the terrain geometry. Including tracer mass transport naturally extends the work of Leukauf et al. (2015), which focuses on the time of the inversion breakup. The inclusion of different initial stability conditions also extends the parameter range. By dividing the valley atmosphere into sub-volumes representing the CBL, the slope wind layer and the stable core, we can calculate tracer mass fluxes between these volumes for a large number of stability/forcing combinations.

This study is organized as follows: in Sect. 2, the model set-up and definitions of different volumes within the valley atmosphere are presented. The general evolution of the flow and the tracer distribution is described in Sect. 3.1, the bulk fluxes between volumes are presented in Sect. 3.2 and the total tracer mass transport is shown in Sect. 3.3. We describe the dependence of the tracer mass fluxes on the forcing and the initial atmospheric stability in Sect. 3.4 and introduce a breakup-parameter in Sect. 3.5. The findings are discussed in Sect. 4 and conclusions are drawn in Sect. 5.

\section{Methods}

\subsection{Model set-up}

We use the Weather Research and Forecasting model (WRFARW), version 3.4. This model has already been successfully used for simulations of thermally driven winds in a previous study by Leukauf et al. (2015) and by other authors both with a kilometre-scale resolution and for large eddy simulations (LES) (Catalano and Cenedese, 2010; Wagner et al., 2014, 2015a, b).
The model configuration is similar to the one in Leukauf et al. (2015), with only a few differences regarding terrain geometry, domain size, grid resolution, forcing and initial stratification. The chosen model topography is a simplified version of the one used by Schmidli (2013), which resembles a broad Alpine valley with a crest height of $1500 \mathrm{~m}$ and was also used in other studies (e.g. Wagner et al., 2014; Leukauf et al., 2015). The depth of the valley is similar to the Inn Valley, Austria, while its width matches approximately the section of the Rhine Valley between Switzerland and Austria close to Lake of Constance. In this study, we focus on the impact of stability and forcing strength; therefore, we decided to replace the sine-shaped slopes with linearly rising slopes to avoid flow features introduced by a changing slope angle. The topography used in this study is displayed in Fig. 1. It consists of a $4 \mathrm{~km}$ broad valley bottom, flanked by two slopes of constant slope angle rising over a width of $6 \mathrm{~km}$ to the crest at $1500 \mathrm{~m}$ relative to the valley floor. The crest on each side is characterized by a $500 \mathrm{~m}$ wide plateau. In the horizontal, the domain consists of $340 \times 200$ grid points. With a mesh size of $50 \mathrm{~m}$, this leads to a $17 \mathrm{~km}$ wide and $10 \mathrm{~km}$ long domain. The $x$ axis is cross-valley oriented and the $y$ axis is parallel to the valley. We use a vertically stretched grid with 119 levels. At the valley bottom, the lowest full model level lies at $12 \mathrm{~m}$ and the second one at $25 \mathrm{~m}$ above the surface, leading to a minimum vertical grid spacing of $\Delta z=13 \mathrm{~m}$. The model level distance increases vertically to a maximum of $75 \mathrm{~m}$ and the topmost model level is located at $8 \mathrm{~km}$ above mean sea level (a.m.s.1.). The upper $2 \mathrm{~km}$ of the domain are occupied by a Rayleigh damping layer with a damping coefficient of $0.003 \mathrm{~s}^{-1}$. The valley floor is at $0 \mathrm{~m}$ a.m.s.l. and periodic boundary conditions are used both in $x$ and $y$ direction.

The surface sensible heat flux is prescribed using a sine function with amplitudes $A_{\mathrm{shf}}=62.5,125,250$, and $375 \mathrm{~W} \mathrm{~m}^{-2}$ respectively, a period of $24 \mathrm{~h}$ and zero crossings at 06:00 and 18:00 local time (LT). This set-up mimics sunrise and sunset at 06:00 and 18:00 LT respectively. These amplitudes cover the range reported for different sites at an Alpine valley during weak synoptic conditions (Rotach and Zardi, 2007). The model is initialized at 06:00 LT and is run for $12 \mathrm{~h}$; hence, only daytime conditions are considered. Neither a boundary layer parametrization nor a land surface scheme is required. A 1.5-order three-dimensional (3-D) turbulence kinetic energy closure (Deardorff, 1980) is used for the parametrization of sub-grid-scale turbulence and a Monin-Obukhov similarity scheme (Monin and Obukhov, 1954) is applied at the surface.

The model is initialized with idealized soundings characterized by a constant Brunt-Väisälä frequency $N$. For the reference forcing of $A_{\mathrm{shf}}=125 \mathrm{~W} \mathrm{~m}^{-2}, N$ is varied between 0.006 and $0.020^{-1}$ with an increment of $0.002^{-1}$. For the other cases, only four simulations are carried out for each $A_{\text {shf }}$ with $N=0.006,0.010,0.014$, and $0.018^{-1}$. In all cases the surface temperature is $T_{0}=280 \mathrm{~K}$, the surface pressure 

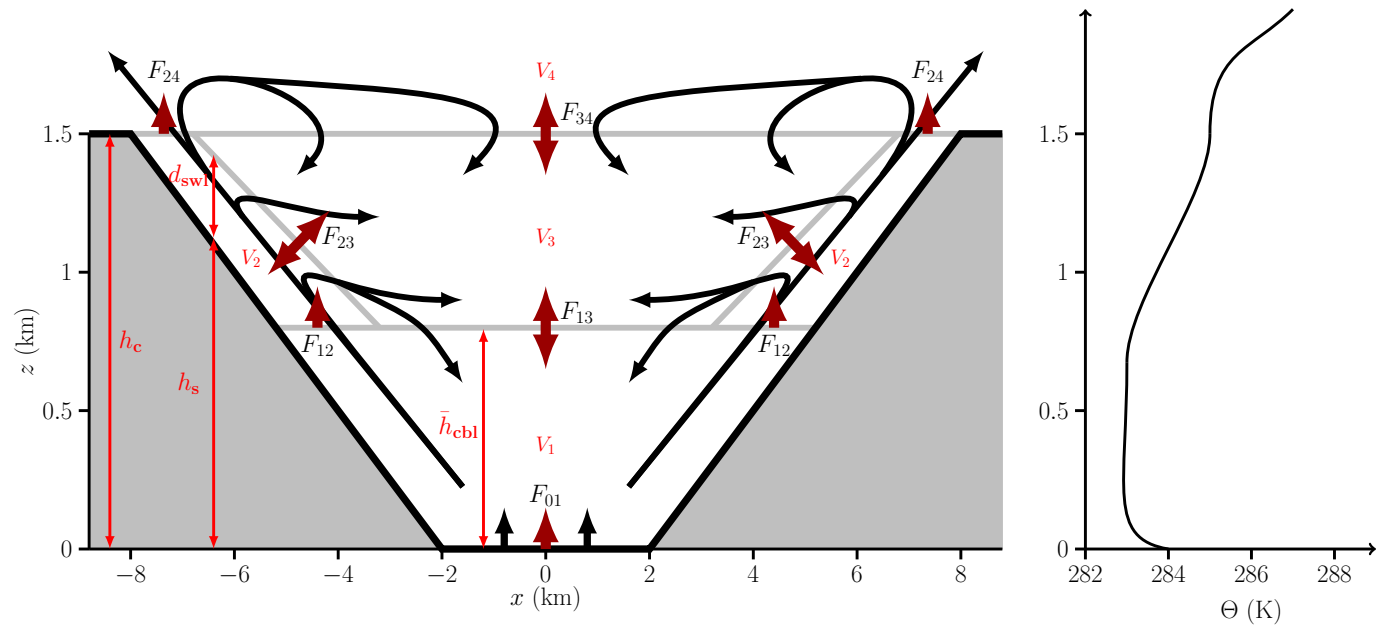

Figure 1. Valley topography with a schematic representation of volumes and tracer mass fluxes and a typical profile of potential temperature at the centre of the valley after a number of hours of model integration. The valley is homogeneous in $y$ direction and is $10 \mathrm{~km}$ long. The valley volume is split into three time-dependent parts (indicated by grey lines): the convective boundary layer ( $\left.V_{1}\right)$, the slope wind layer $\left(V_{2}\right)$, and the stable core $\left(V_{3}\right)$. The volume of the atmosphere above the valley $\left(V_{4}\right)$ is assumed to be time invariant. Notice that $V_{2}$ is the sum of the two-slope wind layers on the two valley sides. Shown is regime 1 for well-defined volumes. See text for explanation of regime 1 and 2. Black arrows illustrate some typical tracer trajectories. Thick red arrows represent bulk tracer mass fluxes between the volumes. Single headed arrows are used for bulk fluxes, which are predominately directed upwards, while double headed arrows represent integrals of local fluxes, which change sign either in time or along the interface between volumes.

Table 1. An overview of different sets of simulations. The reference simulation S1N10 is highlighted using bold typeset. See text for further explanation.

\begin{tabular}{lccc}
\hline Simulation set & Acronyms & $A_{\text {shf }}\left(\mathrm{W} \mathrm{m}^{-2}\right)$ & $N\left(10^{-3} \mathrm{~s}^{-1}\right)$ \\
\hline Half forcing & S0.5N08...S0.5N18 & 62.5 & $6,10,14,18$ \\
Reference forcing & S1N06, S1N08, S1N10...S1N20 & $\mathbf{1 2 5}$ & $6,8, \mathbf{1 0}, \ldots 20$ \\
Double forcing & S2N08..S2N18 & 250 & $6,10,14,18$ \\
Triple forcing & S3N08...S3N18 & 375 & $6,10,14,18$ \\
\hline
\end{tabular}

$p_{0}=1000 \mathrm{hPa}$ at $z=0 \mathrm{~m}$ a.m.s.l., and the relative humidity is $40 \%$ at all levels. The atmosphere is initially at rest. In order to trigger convection, a randomly distributed perturbation of $0.5 \mathrm{~K}$ is added to the initial potential temperature at the lowest five model levels. The simulations are labelled with acronyms such as S1N10, where "S" stands for "sine forcing", the following number is the factor with which the reference forcing amplitude of $125 \mathrm{~W} \mathrm{~m}^{-2}$ is multiplied, $N$ refers to the Brunt-Väisälä frequency and the number afterwards is its value in $10^{-3} \mathrm{~s}^{-1}$. An overview of all simulations is given in Table 1 .

The passive tracer, which is used to measure the horizontal and vertical exchange rates, is released at the lowest model level at every grid point between $x=-2 \mathrm{~km}$ and $+2 \mathrm{~km}$, i.e. at the valley bottom and along the whole length of the valley. The emission rate is set to 1 unit per time step, an arbitrary but constant value. The emission starts immediately at the beginning of the simulation, i.e. at 06:00 LT.

\subsection{Averaging methods}

The procedures used to calculate vertical and horizontal fluxes follow the averaging approach of Schmidli (2013) and are the same as in Leukauf et al. (2015), but applied to tracer mass fluxes.

A variable $\tilde{\phi}$ consists of a component resolved by the model $\bar{\phi}$ and a sub-grid-scale component $\phi^{\prime}$. Averaging the model grid variable $\bar{\phi}$, gives the mean component $\langle\bar{\phi}\rangle$, and subtracting the mean from $\bar{\phi}$ yields the resolved turbulence component (i.e. $\phi^{\prime \prime}=\bar{\phi}-\langle\bar{\phi}\rangle$ ). In total, $\widetilde{\phi}$ is decomposed into mean, resolved turbulence and sub-grid-scale turbulence component:

$\widetilde{\phi}=\langle\bar{\phi}\rangle+\phi^{\prime \prime}+\phi^{\prime}$. 
The averaging $<^{-}>$is hereby defined as an average along the valley and over $T=41 \mathrm{~min}$. This corresponds to the minute of the timestamp plus/minus $20 \mathrm{~min}$; this averaging interval was used in previous studies (e.g. Wagner et al., 2014), where it was demonstrated that at least $30 \mathrm{~min}$ are required to obtain robust averages. In the WRF model, the tracer is defined by the mass mixing ratio $\bar{r}$. Hence the tracer density is $\bar{\rho}_{\text {tr }}=\bar{r} \bar{\rho}$, where $\bar{\rho}$ is the air density. The total vertical tracer mass flux in $z$ direction can then be decomposed into

$$
\underbrace{\left\langle\overline{\widetilde{w} \widetilde{\rho}_{\mathrm{tr}}}\right\rangle}_{\text {TOT }}=\underbrace{\langle\bar{w}\rangle\left\langle\bar{\rho}_{\mathrm{tr}}\right\rangle}_{\text {MEA }}+\underbrace{\left\langle\overline{w^{\prime \prime} \rho_{\mathrm{tr}}^{\prime \prime}}\right\rangle}_{\text {RES }}+\underbrace{\left\langle\overline{w^{\prime} \rho_{\mathrm{tr}}^{\prime}}\right\rangle}_{\mathrm{SGS}} .
$$

The same decomposition can be applied to the total horizontal tracer mass flux $\left\langle\overline{\widetilde{u} \widetilde{\rho}_{\text {tr }}}\right\rangle$.

\subsection{Definition of volumes and fluxes in between}

As long as the valley atmosphere is characterized by at least one stably stratified layer and anabatic slope winds exist, the valley volume can be divided into three different subvolumes (see Fig. 1).

The first volume $V_{1}$ represents the CBL in the lower part of the valley. We define its upper boundary by a horizontally constant height $\bar{b}_{\text {cbl }}$, which is the CBL height averaged over the valley floor. The second volume $V_{2}$ is the sum of the two-slope wind layers on the two valley sides. $V_{2}$ is defined by the depth of the slope wind layer, $d_{\mathrm{swl}}$, the upper boundary at crest height, $h_{\mathrm{c}}$ and the lower boundary at the average CBL height $\bar{h}_{\text {cbl }}$. One might argue that the slope wind layer also exists over the slopes in $V_{1}$. However, it is hard to separate the slope wind layer from the $\mathrm{CBL}$ in this region, so that the chosen definition is more appropriate for quantifying tracer mass fluxes in and out of the slope wind layer. The rest of the valley volume is occupied by the stable core and is denoted by $V_{3}$. Finally, the atmosphere above the valley acts as a fourth volume $V_{4}$. Notice that $\bar{h}_{\mathrm{cbl}}=\bar{h}_{\mathrm{cbl}}(t)$ and $d_{\text {swl }}=d_{\text {swl }}(x, t)$. Hence, the volumes $V_{1}, V_{2}$, and $V_{3}$ are time dependent and $V_{4}$ is assumed to be time invariant. At sunrise (06:00 LT), when neither a slope wind layer nor a CBL exists, the whole valley is occupied by $V_{3}$. We call this initial state regime 0 . Figure 1 illustrates the subsequent regime 1 with well-defined $V_{1}, V_{2}$, and $V_{3}$. When the stable core is completely eroded, i.e. the so-called temperature inversion breakup is reached, volumes $V_{2}$ and $V_{3}$ become zero and the valley is completely occupied by $V_{1}$. The upper boundary of $V_{1}$ is in this case $h_{\mathrm{c}}$. We denote this state by regime 2 . This regime is reached when $\bar{h}_{\text {cbl }}$, the horizontally averaged CBL height, is detected at or above crest height. The CBL height and the slope wind layer depth are defined in Sect. 2.4.

The total tracer mass flux integrated spatially over the interface between two volumes is called bulk flux and is denoted by $F_{i j}$, with the two indices $i$ and $j$ referring to the two volumes between which the flux is defined (see Fig. 1). A flux directed from $V_{i}$ to $V_{j}$ is counted positive. All bulk fluxes are defined to be normal to their respective boundaries. Since the valley geometry and the corresponding slope flow circulation is symmetric with respect to the valley centre, we treat the two-slope wind layers as a single volume $V_{2}$. Hence, $F_{23}$ represents the sum of the two bulk fluxes from the twoslope winds layers into $V_{3}$ or vice versa. Finally, the spatial integral of the prescribed constant surface tracer mass flux at the bottom of the valley is denoted as $F_{01}$. It is useful to normalize fluxes between volumes with the flux at the surface, i.e. $f_{i j}=F_{i j} / F_{01}$. By quantifying tracer mass fluxes between volumes as relative fluxes $f_{i j}$, the results are independent of the strength of the surface emission rate $F_{01}$. The integral of the bulk flux over the duration of the simulation $(12 \mathrm{~h})$ reveals the mass passing the border between two volumes, which is called the total transport and is denoted by $M_{i j}$. Correspondingly, the total tracer mass released at the surface is $M_{01}$ and the normalized tracer mass crossing the interface between volumes $i$ and $j$ is $m_{i j}=M_{i j} / M_{01}$. Due to the averaging operations which are performed along the $y$ axis and over $41 \mathrm{~min}$, the first point in time when full averages are available is 06:45 LT. The integral over time is based on model output stored every $5 \mathrm{~min}$.

Vertical bulk tracer mass fluxes are calculated by integrating the total tracer mass flux $\left\langle\widetilde{\widetilde{w} \widetilde{\rho_{\text {tr }}}}\right\rangle$ (see Eq. 2) along the border between the respective volumes. It is important to keep in mind that the length and position of the borders between volumes change over time as the boundary layer evolves. Hence, the volumes $V_{1}$ to $V_{3}$ are not constant in time, which leads to tracer mass tendencies due to the change of the respective volume size. Since the convective boundary layer grows during the day, tracer mass is re-incorporated into $V_{1}$ by this process, whereas $V_{2}$ and $V_{3}$ lose some tracer mass this way. This is a relatively slow, but relevant process. This aspect has to be considered when comparing budgets and fluxes between different points in time. Since the total tracer mass flux is calculated on full model levels, an interpolation is necessary to calculate correct fluxes between two grid cells. For vertical fluxes, this interpolation must be done only between two grid cells located above each other, while for horizontal fluxes, the change in height due to terrain following coordinates has to be taken into account. The interpolation is error prone when applied to a region where fluxes have strong gradients. This is especially the case for $F_{23}$, for which the intersection between $V_{2}$ and $V_{3}$ passes right through an area with strong horizontal flux gradients. For this reason it is necessary to calculate $F_{23}$ indirectly. Assuming that the vertical fluxes and the tracer mass tendencies are exact, we can invert the tracer mass budget equation for either $V_{2}$ or $V_{3}$ to obtain $F_{23}$. The equation for $V_{2}$ is

$$
F_{23}=\underbrace{L_{y} \int_{L_{12}}\left\langle\overline{\widetilde{w} \widetilde{\rho_{\text {tr }}}}\right\rangle \mathrm{d} x-L_{y} \underbrace{}_{F_{24}}\left\langle\widetilde{\widetilde{w} \widetilde{\rho_{\text {tr }}}}\right\rangle \mathrm{d} x}_{F_{12}}-L_{L_{24}} \int_{A_{2}} \frac{\partial\left\langle\bar{\rho}_{\mathrm{tr}}\right\rangle}{\partial t} \mathrm{~d} A,
$$


and a similar equation holds for $V_{3}$ :

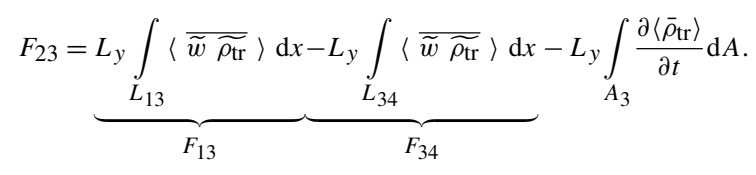

Here, $A_{i}$ is the vertical side face of $V_{i}$ on the $x-z$ plane and $L_{i j}$ the borderline between $V_{i}$ and $V_{j}$ in $x$ direction. The local tendencies in Eqs. (3) and (4) are integrated only over cross sections on the $x-z$ plane since they are already averaged in $y$ direction. However, the multiplication with $L_{y}$, the length of the valley, yields terms representative for the whole volume. The vertical bulk fluxes on the right side of Eqs. (3) and (4) are validated by comparing the volume integrated tracer mass tendencies in $V_{1}$ and $V_{4}$ with the sum of bulk fluxes in and out of these volumes and good agreement is generally found. The calculation of $F_{23}$ is done for $V_{2}$ and $V_{3}$ individually according to Eqs. (3) and (4) respectively. The agreement between these two independent results for $F_{23}$ is satisfying, but the results are not identical. Due to errors caused by interpolation and changing volume sizes, the tracer mass budgets are not perfectly closed, especially in the early morning hours when $V_{1}$ and $V_{2}$ are still relatively small. This causes a difference between the two calculations of $F_{23}$. The difference is largest in the first $2 \mathrm{~h}$ with about $20 \%$ relative to $F_{01}$, but decreases afterwards to nearly zero. Henceforth, we use an average of $F_{23}$ based on the two values from Eqs. (3) and (4) to minimize the effects of errors.

\subsection{Definitions of boundary layer and slope wind layer}

In order to quantify tracer mass fluxes between the volumes described above, working definitions of CBL height $h_{\mathrm{cbl}}$ and slope wind layer depth $d_{\mathrm{swl}}$ are needed. Here, $d_{\mathrm{swl}}$ is the vertical distance between terrain surface $h_{\mathrm{s}}$ and upper boundary of the slope wind layer, whereas the slope wind layer height $h_{\text {swl }}$ is an absolute height, i.e. $h_{\mathrm{swl}}=h_{\mathrm{s}}+d_{\text {swl }}$. Defining the boundary layer height over complex terrain is not straight forward since $h_{\mathrm{cbl}}$ may strongly depend on terrain and synoptic conditions (De Wekker and Kossmann, 2015). Herein, we calculate the average CBL height $\bar{h}_{\mathrm{cbl}}$ above the flat valley floor based on model profiles averaged between $x=-2$ and $x=+2 \mathrm{~km}$ using a criterion described below. The daytime slope wind layer has characteristics similar to a CBL over a flat surface such as the valley floor, with a super-adiabatic surface layer followed by a well-mixed layer and a capping inversion. In other words, the vertical profiles of potential temperature and vertical heat flux of slope wind layer and CBL are very similar. Hence, for determining $h_{\text {swl }}$ we use the same criterion as for $h_{\mathrm{cbl}}$. Consequently, the CBL height over the flat part of the valley will smoothly change into the slope wind layer height at $x= \pm 2 \mathrm{~km}$.

In principle, various definitions of $h_{\mathrm{cbl}}$ and $h_{\mathrm{swl}}$ are conceivable. First, the CBL height can be defined as the lowermost level where the vertical gradient of the mean potential temperature $<\bar{\theta}>$ exceeds the value of $0.001 \mathrm{~K} \mathrm{~m}^{-1}$ when moving upward from the surface. This definition is similar to that of Catalano and Moeng (2010) but without the additional constraint on the heat flux. We shall refer to this definition as the $\theta$-gradient criterion. The classical definition refers to the turbulent nature of the $\mathrm{CBL}$ and defines the CBL height as the level where the total vertical heat flux has its negative minimum (Sullivan et al., 1998). This definition can lead to an artificial step-like structure of the CBL height in space and time when the heat flux minimum is not well defined at a distinct level but is rather spread over a certain altitude range. For this reason, we slightly modify this criterion and determine the CBL height at the level $i$ at which the decrease of the vertical heat flux between levels $i$ and $i+1$ is less than $5 \%$. This criterion is referred to as the heat-flux criterion. As mentioned above, both criteria could be used not only to determine the CBL above the valley floor, but also to identify the slope wind layer depth. Yet another way to define $h_{\mathrm{swl}}$ is to separate the upward flow inside the slope wind layer from the subsidence in the centre of the valley. Here, the slope wind layer is the layer above the slope where the mean vertical wind component $\langle\bar{w}>$ is positive. This definition is implicitly used, e.g. in the analytical model of Vergeiner and Dreiseitl (1987) since it characterizes the upward mass flux that determines export processes. Is is only meaningful above the slopes. We shall refer to this definition as the $w$ criterion.

Boundary layer heights and slope wind layer depths based on these three definitions are shown for simulation S1N10 in Fig. 2. In the morning until noon, the $\theta$-criterion gives lower values of $h_{\mathrm{cbl}}$ above the valley floor than the heat-flux criterion. Over the slopes, the two definitions reveal very similar $d_{\text {swl }}$, with the $\theta$-gradient criterion again yielding the lowest boundary layer height. However, as the stable core is eroded, the CBL height according to the $\theta$-gradient criterion reaches much greater heights than the heat-flux criterion since it is sensitive to the threshold value of $0.001 \mathrm{~K} \mathrm{~m}^{-1}$. This feature occurs quite suddenly once the vertical gradient of the potential temperature falls below this threshold. Choosing a lower threshold would delay but not prevent this behaviour. For this reason, the $\theta$-gradient criterion is not suitable for a weak initial stability and later in the afternoon. The $w$ criterion gives slope wind layer depths which are very similar to those generated with the heat-flux criterion within the slope wind layer.

Since the $w$ criterion cannot yield a suitable CBL height in the centre of the valley and the $\theta$-gradient criterion is sensitive to the chosen threshold is case of a weak static stability, we use the heat-flux criterion in the remainder of the paper to determine both the CBL and $h_{\mathrm{swl}}$. Before the breakup of the inversion (regime 1$), \bar{h}_{\mathrm{cbl}}$ is used as the upper (lower) boundary of $V_{1}\left(V_{2}\right.$ and $\left.V_{3}\right)$ while $h_{\text {swl }}$ serves as the lateral boundary between $V_{2}$ and $V_{3}$ (see Fig. 1). A spatial average using a Hann window over $2 \mathrm{~km}$ is applied to $d_{\mathrm{swl}}$ before calculating $h_{\text {swl }}$ to smooth wiggles which would be unsuitable for the definition of $V_{2}$ and its border to $V_{3}$. Finally, the upper boundary of $V_{2}$ and $V_{3}$ is marked by the crest height $h_{\mathrm{c}}$. 

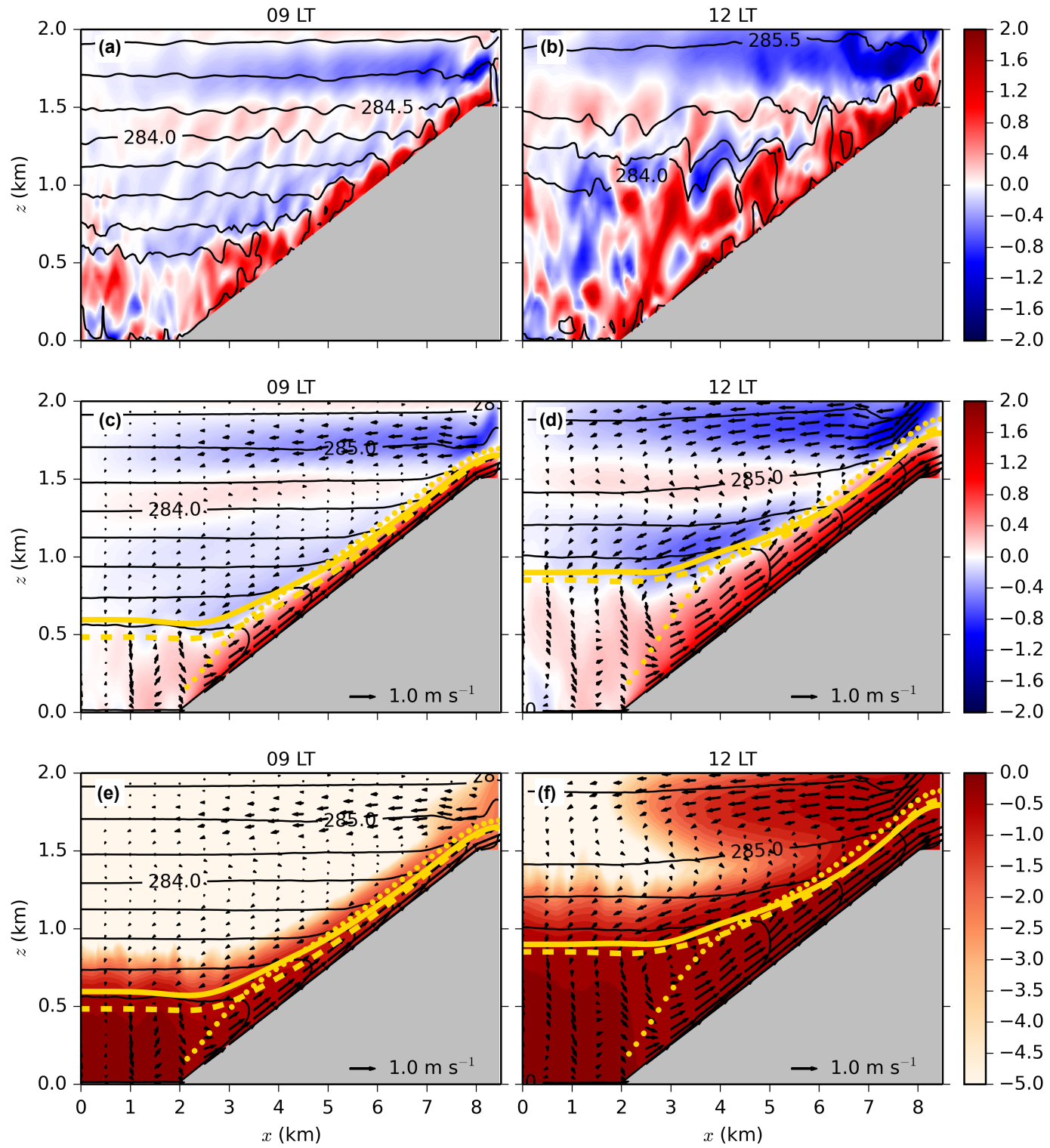

Figure 2. Vertical cross sections at 09:00 LT (left column) and 12:00 LT (right column) for the simulation S1N10. Shown fields in (a) and (b) are instantaneous cross-valley wind component $\left(\mathrm{m} \mathrm{s}^{-1}\right)$ as colour contours and instantaneous potential temperature as black contours with $0.5 \mathrm{~K}$ increment at $y=5 \mathrm{~km}$. The same variables, but averaged over the period $T$ and along the $y$ axis, are shown in (c, d) together with wind vectors based on averaged components. The averaged and normalized tracer mass density is $\operatorname{shown}$ as $\log _{10}\left(\left\langle\bar{\rho}_{\mathrm{tr}}\right\rangle / \max \left(\left\langle\bar{\rho}_{\mathrm{tr}}\right\rangle\right)\right)$ with colour contours in (e, f), along with potential temperature and wind vectors as in (c, $\mathbf{d})$. The three different boundary layer and slope wind layer heights are shown as yellow lines. They are based on the heat-flux criterion (solid line), the $\theta$-gradient criterion (dashed line), and the $w$ criterion (dotted line). See text for further explanation.

After the breakup (regime 2), $V_{2}$ and $V_{3}$ become zero and the upper boundary of $V_{1}$ becomes $h_{\mathrm{c}}$ and remains at this height.

\section{Results}

\subsection{General flow and tracer distribution}

The general flow and tracer distribution are described in this section for the S1N10 simulation, which serves as a reference. The evolution of the other simulations is discussed briefly at the end of this section. 
At the start of the simulation (06:00 LT), the whole atmosphere is stably stratified and characterized by a constant Brunt-Väisälä frequency (regime 0). With the onset of the surface-layer heating, a shallow CBL begins to form in the centre of the valley and a slope wind layer develops (regime 1, Fig. 2a). The turbulent nature of the slope wind layer leads to mixing at its top, a process which leads to its growth (Fig. 2b), but also to exchange of air with the stable core. Averaging in time and along the $y$ axis reveals not only a mean up-slope flow and the recirculation above the valley, but also the formation of a $\mathrm{HI}$ just above the CBL height, next to the lower end of $V_{2}$ (Fig. 2c). At 09:00 LT, the slope wind layer has a depth of $270 \mathrm{~m}$ at $x=4 \mathrm{~km}$ and $140 \mathrm{~m}$ at $x=6 \mathrm{~km}$, i.e. decreases with increasing height. The average wind speed increases from $0.65 \mathrm{~m} \mathrm{~s}^{-1}$ at $x=4 \mathrm{~km}$ to $0.75 \mathrm{~m} \mathrm{~s}^{-1}$ at $x=6 \mathrm{~km}$ respectively. However, the increasing wind speed with height cannot compensate the decreasing $d_{\text {swl }}$, which leads to along-slope convergence. Due to mass continuity, this convergence leads to an export of air from the slope wind layer to the valley centre and, hence, the formation of a HI. The HI is about $300 \mathrm{~m}$ thick perpendicular to the slope, and has a wind speed maximum of about $0.3 \mathrm{~m} \mathrm{~s}^{-1}$. The reduction of $d_{\mathrm{swl}}$ with height is much stronger at 12:00 LT, as indicated by the slope wind layer depths of 525 and $270 \mathrm{~m}$ at $x=4$ and $6 \mathrm{~km}$ respectively. The average wind speed within the slope wind layer has increased only slightly to 0.82 and $0.92 \mathrm{~m} \mathrm{~s}^{-1}$ at the two locations, which leads to a stronger reduction of the along-slope mass flux and therefore to a stronger HI. It is characterized by a mean horizontal wind speed maximum of about $0.6 \mathrm{~m} \mathrm{~s}^{-1}$ (Fig. 2d).

Tracer released at the valley bottom is distributed homogeneously within the CBL and is also advected upwards by slope winds (Fig. 2e). In simulation S1N10, the tracer reaches the crest height about $2.5 \mathrm{~h}$ after the simulation has started. Tracer mass export from $V_{2}$ to the centre of the valley is strongest in the lower part of the stable core $\left(V_{3}\right)$ where the mass flux reduction in the slope wind layer is also strongest. It is noteworthy, that no well-defined horizontal banners of tracer develop within the valley as observed for example in the Inn Valley (Harnisch and Gohm, 2009), but rather a deep layer of tracer without clear separation from the CBL underneath. This is due to the vertically constant initial stability $N$, which we have chosen for this idealized approach. An elevated inversion, much higher than $h_{\mathrm{cbl}}$, would promote the formation of a tracer banner. The HI, being a phenomenon caused by the interaction of the slope wind layer with the stable core, forms as soon as the slope wind layer develops and persists as long as the stable core and the upslope flow exist. This persistence allows the HI to redistribute considerable amounts of tracer mass despite the relatively low mean horizontal wind speed, which ranges between 0.5 and $1.0 \mathrm{~m} \mathrm{~s}^{-1}$. In addition to the mean advection by the HI, the tracer is transported to the stable core by turbulent mixing at the top of the CBL above the valley floor as well as at the top of the slope wind layer.
As the boundary layer evolves, the slope wind layer grows in depth and the wind speed within the HI increases (cf. Fig. 2c and d). At 12:00 LT, tracer mass, which has reached the atmosphere above the valley, is distributed in an almost neutral layer which develops due to a second and stronger circulation above the valley. This circulation is ultimately responsible for reimporting tracer mass through the valley top into the stable core (Fig. 2f). With continuous heating, the valley atmosphere would eventually become completely neutral and reach regime 2. However, the reference case S1N10 has a forcing strength slightly too weak for the breakup, but cases with a stronger forcing or a weaker stratification do reach this regime. If this is the case, the CBL inside and the neutral layer above the valley merge, creating a deeper CBL that extends beyond crest height. Within this layer, turbulent mixing is very effective and with a constant source at the bottom, this leads to large vertical tracer mass fluxes at crest height.

The tracer mass fluxes responsible for the redistribution of tracer mass are shown in Fig. 3 for S1N10 at 12:00 LT. Tracer mass is transported along the slopes primarily by mean horizontal and vertical fluxes (Fig. 3a, b). At the border of $V_{2}$, defined by $d_{\text {swl }}$, strong horizontal fluxes carry tracer mass towards the valley centre while weaker downward fluxes indicate a partial recirculation of tracer mass into the $\mathrm{CBL}\left(V_{1}\right)$. Horizontal transport due to the circulation above the valley is also indicated by a strong mean tracer mass flux. Overall, the horizontally resolved turbulent flux is 1 order of magnitude smaller than the corresponding mean flux (cf. Fig. 3a and c). The vertical resolved turbulent flux is positive in the CBL and $V_{2}$ and strongest in magnitude in the centre of the valley (Fig. 3d). Over the slopes, the vertical-resolved turbulent flux is, again, 1 order of magnitude smaller than the mean vertical tracer mass flux, but extends further up into the stable core (Fig. $3 b$ and d).

Simulations with a stronger forcing or a weaker stability than the reference case S1N10 exhibit a similar evolution, but the inversion breakup (regime 2) is reached. In the case of a weaker forcing or a stronger stability than S1N10, the breakup does not occur. For example, the breakup is reached after about $4 \mathrm{~h}$ for S1N06, thus allowing for a larger amount of tracer export from the valley atmosphere, whereas for simulations S1N12 to S1N20 the breakup is never reached, which restricts vertical venting considerably. All simulations exhibit a similar pattern of tracer mass fluxes as long as the breakup is not reached. However, for a stronger stratification, the slope wind layer is shallower and the CBL grows more slowly. The tracer mass flux at the top of the slope wind layer is weaker and the recirculation within the valley is more pronounced. Only a single HI develops in all cases.

\subsection{Bulk tracer mass fluxes}

Figure 4 shows the normalized bulk fluxes $f_{i j}$ as a function of time for the simulations S1N06 to S1N12 and S1N16. In 

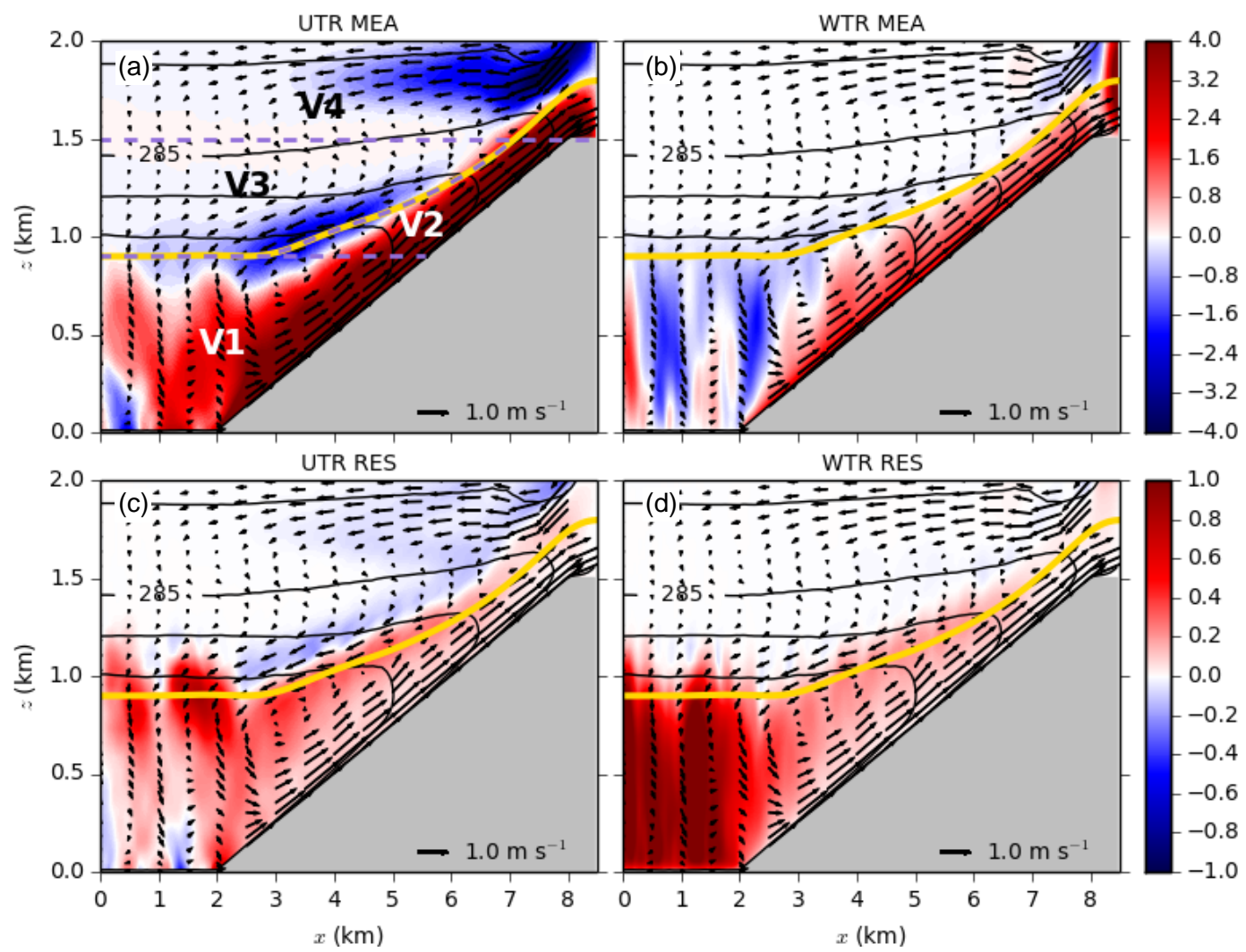

Figure 3. Horizontal and vertical tracer mass fluxes for simulation S1N10 at 12:00 LT. Shown are (a) cross-valley mean (UTR MEA), (b) vertical mean (WTR MEA), (c) cross-valley turbulent resolved (UTR RES), and (d) vertical turbulent resolved (WTR RES) tracer mass fluxes as colour contours. All fluxes are normalized by the tracer mass flux at the surface and are therefore dimensionless. The colour bars to the right apply to each row. Notice that the colour scale in $(\mathbf{c}, \mathbf{d})$ is smaller by a factor of 4 than in $(\mathbf{a}, \mathbf{b})$. Potential temperature, wind vectors and the boundary/slope wind layer height according to the heat-flux criterion are as in Fig. 2. The volumes V1 to V4 are also shown in (a). The purple dashed lines denote the boundaries between these volumes.

the early morning, both fluxes out of $V_{1}$ are positive where $f_{13}$ is larger than $f_{12}$. The bulk flux from $V_{1}$ into the $V_{2}$ is zero in the beginning but grows steadily as the slope winds intensify with increasing surface sensible heat flux. The flux between $V_{3}$ and $V_{2}$ is negative, which means that it is directed towards $V_{2}$ and advects some of the tracer brought into $V_{3}$ by $f_{13}$. This happens since the CBL is still very shallow $(<500 \mathrm{~m})$ and air enters $V_{2}$ at such low levels not only vertically but also horizontally. The evolution of the fluxes in all S1 simulations is very similar until 09:00 LT.

At about 08:30 LT the tracer reaches crest height and is exported out of the valley. This is indicated by an increasing flux $f_{24}$ and happens for all $\mathrm{S} 1$ simulations, but its maximum depends strongly on the initial stratification. In the case of the weakest stratification (N06) this flux reaches about $50 \%$ before the breakup. In this case, not only $f_{24}$ is positive, but also $f_{34}$, which indicates that even in the centre of the valley more tracer is exported than reimported at crest height. The tracer mass export out of $V_{2}$ increases more slowly the stronger the initial stratification is and much lower peak values are reached. For example, in S1N08, $f_{24}$ has a peak value of $100 \%$ at noon while for S1N16 this flux has a maximum of only about $25 \%$. If the inflow of tracer mass at the lower boundary of $V_{2}$ is greater than the outflow at crest height, tracer mass is advected towards the stable core. For strong initial stratifications (i.e. N12 and stronger), $f_{24}$ is always smaller than $f_{12}$. For these simulations, $f_{23}$ is the main source of tracer mass for the stable core.

In the afternoon, simulations differ greatly from each other depending on the initial stability. While simulations with a weak stability reach the breakup either before noon (S1N06, Fig. 4a) or in the early afternoon (S1N08, Fig. 4c), this does not happen for a stronger initial stability (Fig. 4d, e, f). In regime 2 , the only remaining flux is $f_{14}$, which climbs to values of about $100 \%$. For the weakest stability, it oscillates in the afternoon between 60 and $125 \%$. The reason for these strong oscillations are large convective cells, which develop in the boundary layer and redistribute tracer mass vertically. If the valley would be longer, the averaging area would be larger and those oscillations would presumably be much smaller. 

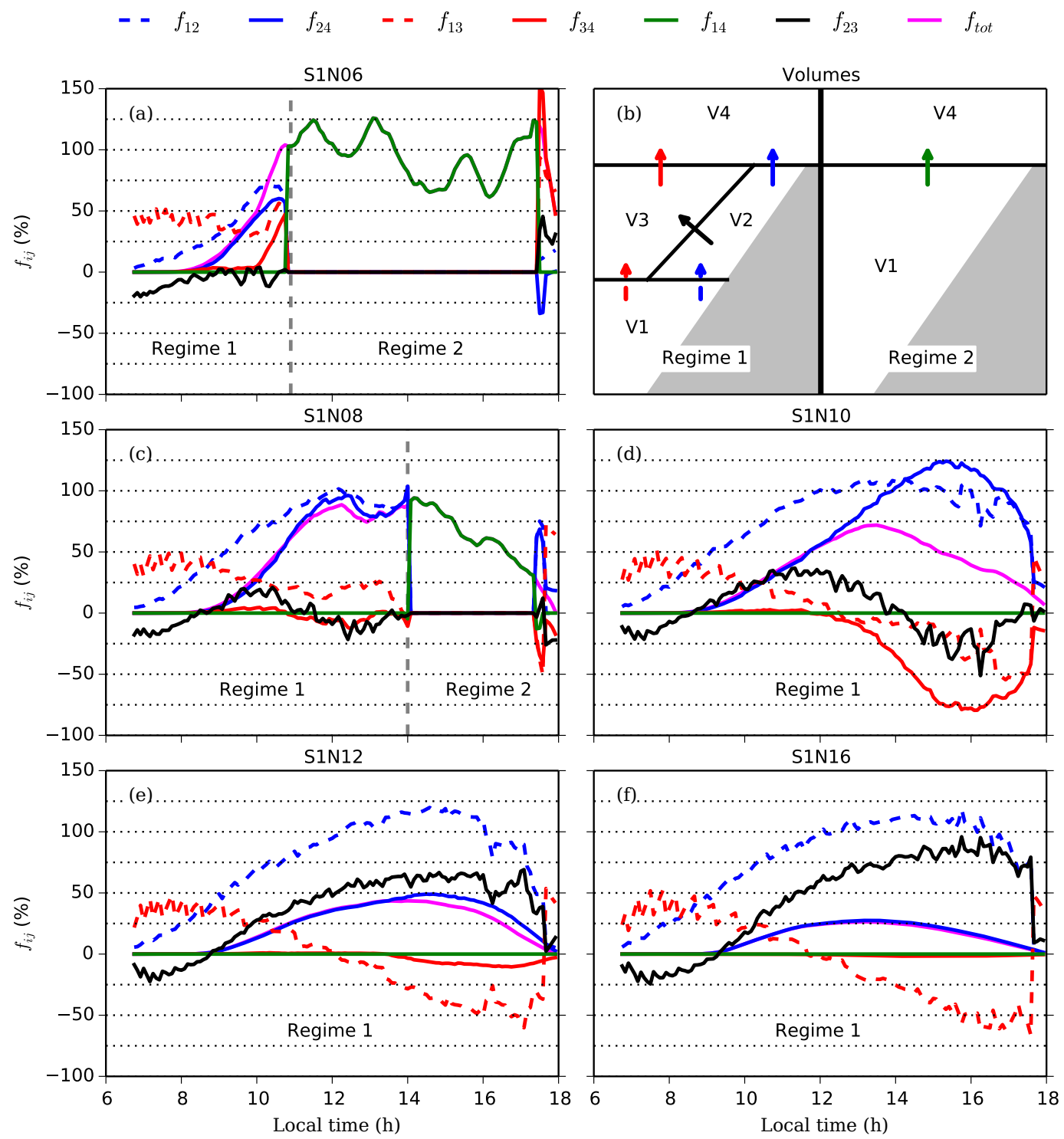

Figure 4. Normalized bulk tracer mass flux for a set of S1 simulations with five different values of initial static stability: (a) N06, (c) N08, (d) N10, (e) N12, and (f) N16. The tracer mass fluxes are integrated at the boundaries between different volumes and normalized by the spatially integrated tracer mass flux at the surface $F_{01}$. The sketch in (b) shows the position of different volumes and associated bulk fluxes. The left side represents a valley atmosphere before the breakup (regime 1), whereas the right side shows the situation after the breakup (regime 2). A grey dashed line indicates the change from regime 1 to 2 . Colours of arrows in (b) are the same as the colours of the corresponding lines in the other panels, whereby solid (dashed) arrow shafts correspond to solid (dashed) lines.

For the case S1N10, the forcing is too weak to completely remove the inversion which leads to a large maximum export of tracer mass but it grows more slowly compared to simulations with a weaker initial stratification. Due to this slower increase, more tracer mass accumulates before noon in $V_{1}$. Eventually, a volume of heavily polluted air will enter $V_{2}$, be advected along the slope and leave the valley at crest height. This leads to peak values for $f_{24}$ of $125 \%$. This flux decreases again once the particularly tracer-rich air has left the valley. Since the inversion is never completely eroded for
S1N10, thermals at the centre of the valley do not reach out of the valley. Instead, tracer mass is reimported at crest height by subsidence. The tracer mass cannot leave the vicinity of the valley due to the periodic boundary conditions; therefore the reimport of tracer mass is very large and reaches a value of $-75 \%$ in the late afternoon. The net export of tracer mass peaks at about $45 \%$ at 14:00 LT. Some of this reimported tracer is again reintroduced into $V_{2}$ below peak height leading to a negative $f_{23}$ (Fig. $4 \mathrm{~d}$ ). For an even stronger initial stability, the subsidence is smaller and the amount of tracer 
mass exported is greatly reduced. Hence, hardly any tracer mass is reimported into the valley volume. The tracer mass flux is instead dominated by the inflow into $V_{2}$ and the outflow from $V_{2}$ into $V_{3}$. This outflow, $f_{23}$, has an amplitude up to 3 times larger than $f_{24}$, the outflow from $V_{2}$ to the atmosphere above the valley. However, the tracer mass advected into the stable core remains only partially in this volume as $f_{13}$ turns negative in the afternoon and reaches values of $-50 \%$. Hence, tracer mass is advected back into $V_{1}$, a flux pattern that nicely illustrates the circular motion of tracer inside the "pollution trap". The efficiency of this circulation increases with increasing static stability $N$. For the strongest stability shown in Fig. 4f, the peak value of net tracer export $\left(f_{24}+f_{34}\right)$ in the early afternoon is only about $25 \%$. The fluxes in the simulations with the most stable initial conditions, S1N14 to S1N20, are very similar, therefore only S1N16 is shown in Fig. 4f.

In the last half hour before sunset (18:00 LT), $V_{1}$ shrinks quickly as the lack of heating from the surface leads to much lower CBL heights and slope wind layer depths. The same happens to $V_{2}$ if this volume is still defined, i.e. when the breakup is never reached. Otherwise, the volumes $V_{2}$ and $V_{3}$ are re-established (Fig. 4a, c). This leads to sharp changes in associated fluxes. This sudden change in volume sizes depends to some degree on the criterion used to define the volumes.

\subsection{Total tracer mass transport}

The total tracer mass $M_{i j}$ transported between $V_{i}$ and $V_{j}$ between 06:00 and 18:00 LT is defined as the integral of $F_{i j}$ over the course of the simulation. Notice that the volumes are not constant in time. This is the reason why the total mass transport does not allow for conclusions on the concentration of pollutants, but is a measure of the average strength of the respective fluxes. Figure 5 shows $M_{i j}$ normalized by the total mass released at the surface, $m_{i j}=M_{i j} / M_{01}$, for simulations S1N06 to S1N20. Overall, it becomes clear that the vertical transport out of $V_{1}$ is strong for all stability conditions, as about $75 \%$ of the tracer mass is either transported into $V_{2}$ $\left(m_{12}\right)$, directly into the stable core $\left(m_{13}\right)$, or out of the valley atmosphere $\left(m_{14}\right)$. The latter happens due to a weak stability after the breakup of the stable stratification in the valley. As $f_{13}$ is directed upwards in the morning and downwards in the afternoon, the net transport directly from $V_{1}$ to $V_{3}$ is relatively small. For the two cases with the weakest stratifications (S1N06 and S1N08), $f_{13}$ is no longer defined after the breakup, so that the total mass transported from $V_{1}$ to $V_{3}$ $\left(m_{13}\right)$ remains positive but, at $20 \%$, relatively small.

Once the tracer mass is in $V_{2}$, the atmospheric stability strongly controls where it will be subsequently transported to. For weaker stability, the tracer mass is predominately exported out of the valley, i.e. large $m_{24}$ and/or $m_{14}$. In the case of N06, about $70 \%$ of the tracer mass released at the surface is exported. This number drops to about 15 to $20 \%$ for N14

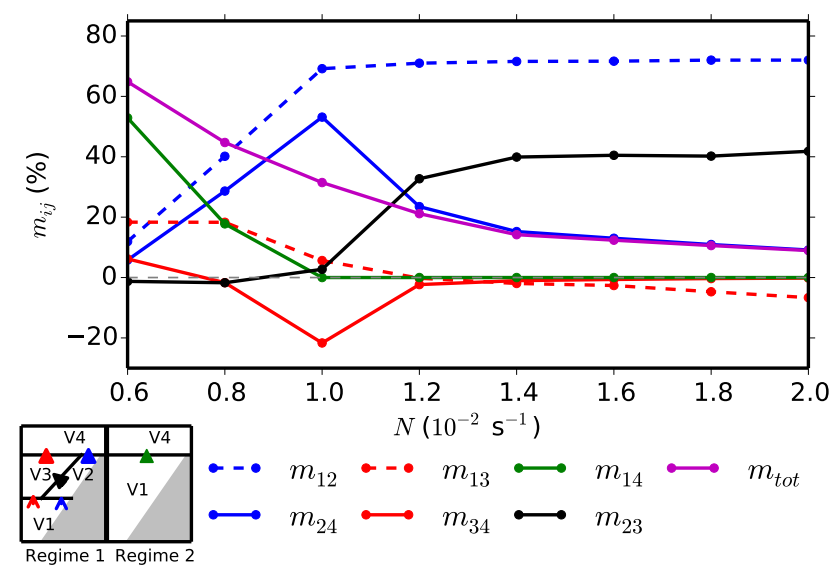

Figure 5. The total mass of tracer passing each interface between two volumes $V_{i}$ and $V_{j}$ and between 06:00 and 18:00 LT, normalized by the total mass of tracer released at the valley floor, i.e. $m_{i j}=M_{i j} / M_{01}$ as a function of stability for the $\mathrm{S} 1$ forcing. The total exported tracer mass over the course of the simulation is $m_{\text {tot }}=m_{24}+m_{34}+m_{14}$. The bottom left is similar to Fig. $4 \mathrm{~b}$, except that the open arrowhead corresponds here to dashed lines.

to $\mathrm{N} 20$, while the mass transport from $V_{2}$ to the stable core increases continuously with increasing stability and reaches values as large as $42 \%$. Before the breakup, tracer mass is exported by the slope winds $\left(m_{24}\right)$ and partially reimported by compensating subsidence near the centre of the valley $\left(m_{34}\right)$. In the case of strong stability, hardly any tracer mass is reimported since there is little mass above the valley that could be reimported by subsidence. For a weak stability, the fluxes associated with export and reimport are combined in $f_{14}$ once the breakup is reached. Since some tracer mass must be accumulated in $V_{4}$ to allow a significant reimport of tracer mass, $m_{34}$ is close to zero for S1N06 and S1N08. Only in the case of S1N10 is a significant amount $(25 \%)$ reimported via $m_{34}$ as this is a simulation with a stability weak enough to allow for a substantial export of tracer, but strong enough to avoid the breakup, so that $V_{3}$ exists even in the late afternoon.

\subsection{Dependence on forcing amplitude and initial stability}

The time of the inversion breakup and, consequently, whether it is reached at all before sunset is an important parameter in describing the tracer mass fluxes between the various valley volumes. This time is important since the exchange with the atmosphere above the valley increases as the valley atmosphere becomes neutral. Since the breakup time strongly depends on initial stability and forcing amplitude, we study the impact of these parameters here in more detail. The overall structure of the valley atmosphere and the magnitudes of tracer mass fluxes between various volumes of the reference simulation are very similar to those from runs with a weaker or stronger forcing. Due to these similarities, we restrict the 
following analysis to the fluxes at the top and the bottom of $V_{2}$.

Figure $6 \mathrm{a}$ shows the evolution of $f_{12}$ for $\mathrm{S} 0.5 \mathrm{~N} 10$ to S3N10 and S1N06 to S1N18 respectively. The magnitude of the vertical flux into $V_{2}$ and the increase in time primarily depends on the forcing strength. Simulations with different stability conditions show a very similar evolution. This can be understood by considering the mass flux along the slope wind layer following the simple analytical slope-flow model of Vergeiner (Vergeiner, 1982; Vergeiner and Dreiseitl, 1987). According to this model, the mass flux $V \delta$ is:

$V \delta=\frac{\frac{H}{\tan (\gamma)}(1-Q)}{c_{\mathrm{p}} \rho \frac{\mathrm{d} \Theta}{\mathrm{d} z}}$,

where $V$ is the slope-parallel wind speed averaged over the depth of this layer, $\delta$ the thickness of the slope wind layer (i.e. its depth measured normal to the slope), $(1-Q) H$ is the fraction of the surface sensible heat flux, which heats the slope wind layer, $\gamma$ is the slope angle, $\frac{\mathrm{d} \Theta}{\mathrm{d} z}$ is the potential temperature gradient in the centre of the valley as a function of $z, \rho$ the air density, and $c_{\mathrm{p}}$ the specific capacity of heat at constant pressure. The stability at CBL-height is similar in all simulations, which explains the rather weak dependence on initial stratification (Fig. 6). In contrast, a stronger forcing leads to a stronger tracer mass flux. The time of the breakup varies between 07:45 LT (S3N06, not shown) and no breakup.

For the export of tracer mass out of $V_{2}$ into the atmosphere above the valley, both stability and forcing are important factors. A stronger forcing results in a stronger up-slope flow and, hence, larger vertical flux $f_{24}$ (see Fig. 6b). Note that the decrease of tracer export is related to the decrease of forcing amplitude in a non-linear fashion. While S1N10 has a peak magnitude of about $125 \%$ at 15:00 LT, S0.5N10 peaks at merely $25 \%$. A similar reduction of peak export $f_{24}$ also results if the stability is increased significantly beyond the critical value that enables the breakup (cf. S1N10 and S1N14). Interestingly, simulation S1N10 has the largest peak flux out of $V_{2}$, even when compared to simulations with a stronger forcing or a weaker initial stability. The reason is the accumulation of tracer mass in $V_{1}$ during the day, due to the fact that the breakup is not reached. In combination with relatively strong slope winds, this leads to a peak export of $125 \%$ once tracer-rich air reaches crest height. For S2N10 and S3N10 this peak value is only about $100 \%$. The export out of $V_{2}$ for N1N06 peaks at about $60 \%$ since the simulation reaches the breakup point and the export is henceforth part of $f_{14}$. It is obvious that simulations with a strong inflow of tracer at the lower end of $V_{2}$ but a weak outflow at the top are characterized by a strong outflow of tracer mass into the stable core (not shown).

The dependence of the total tracer mass transport between $V_{i}$ and $V_{j}$ on the forcing amplitude $A_{\text {shf }}$ is shown in Fig. 7 for N10 and N18. For the weaker stability, N10 (Fig. 7a), the total mass transport between $V_{2}$ and $V_{3}$ is zero, except for the
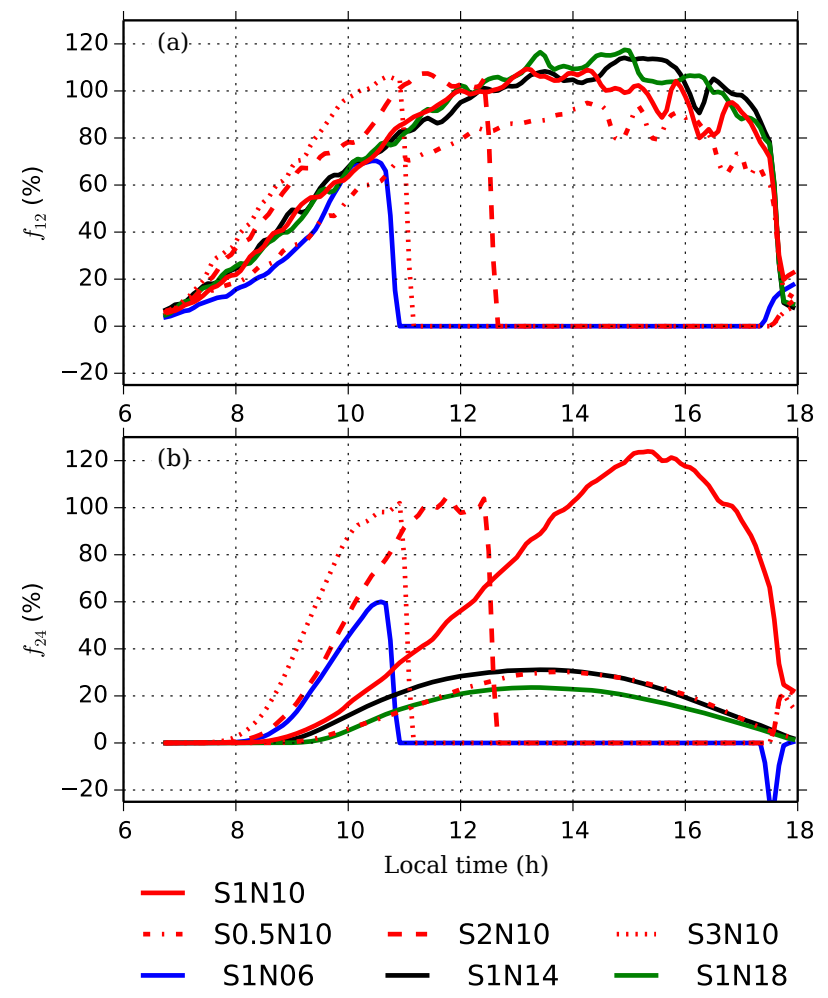

Figure 6. Tracer mass flux (a) $f_{12}$ and (b) $f_{24}$ for N10 and four different forcing amplitudes (S0.5N10, S1N10, S2N10, S3N10) as well as for S1 and four different values of stability (S1N6, S1N10, S1N14, S1N18).

weakest forcing S0.5. The import of tracer mass into $V_{2}$ is slightly higher than the export at crest height with the difference remaining inside $V_{2}$ resulting in a zero net transport to the stable core. This is not the case for the weakest forcing, in which the tracer inflow at the lower end of $V_{2}$ is about $60 \%$ but the outflow only about $15 \%$. Consequently, tracer mass is redirected toward the stable core, with about $30 \%$ and the rest, about $15 \%$, remains in $V_{2}$. The tracer transport from the CBL directly into the stable core $\left(m_{13}\right)$ is rather small and depends only weakly on the forcing.

In the case of the stronger stability, N18 (Fig. 7b), the inflow of tracer into $V_{2}$ is always greater than the outflow, which leads to an horizontal transport towards the stable core on the order of $40 \%$. This flux increases with increasing forcing except for the strongest forcing (S3), where a much stronger vertical outflow at crest height leads to a weaker total flux between $V_{2}$ and $V_{3}$. There is also a recirculation from $V_{4}$ to $V_{3}$ and then again into $V_{2}$ close to crest height. This recirculation implies a negative local flux from $V_{2}$ to $V_{3}$ near crest level and, hence, reduces the bulk flux $m_{23}$. The direct transport of tracer between the CBL $V_{1}$ and the stable core $V_{3}$ is negative for this stability with values reaching $-12 \%$. This is a sign of an effective recirculation at boundary layer height, i.e. part of the tracer that is exported from the CBl 


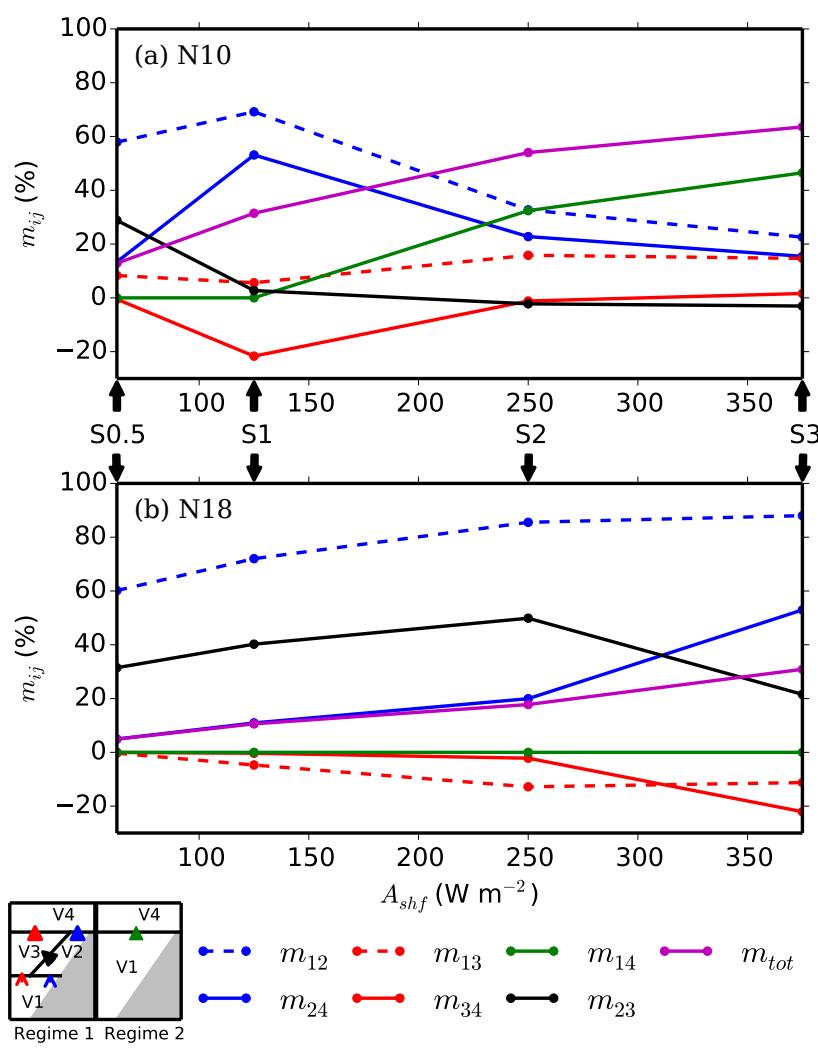

Figure 7. As in Fig. 5, but as a function of the forcing amplitude, $A_{\text {shf }}$, for (a) N10 and (b) N18.

by slope winds is imported again into $V_{1}$ by turbulent mixing and the low level recirculation within the "pollution trap".

The total export of tracer mass $\left(m_{\text {tot }}\right)$ increases almost linearly with the forcing for N18. For N10 this increase is stronger for low-forcing amplitudes but flattens for stronger forcing. Between 13 and $64 \%$ of the tracer mass is exported for N10 but only between 5 and $31 \%$ is exported for N18.

\subsection{A non-dimensional breakup parameter}

It is clear from the previous analysis, that the breakup time is an important timescale for the evolution of the valley boundary layer, which depends on both the initial stability and the forcing amplitude. Hence, the venting of a valley strongly depends in a non-linear way on both parameters, but the total export of tracer mass at crest height may be similar for certain combinations of forcing and stability. A run with a strong forcing and a strong stability exports about as much tracer as a run with a weaker forcing and a weaker stability (Fig. 8a). The question arises whether a common parameter can be found that combines the effect of stability and forcing on the exchange of tracer. The answer will be given below.

After Whiteman and McKee (1982), the breakup time of a valley inversion primarily depends on $Q_{\text {req }}$, the energy re-

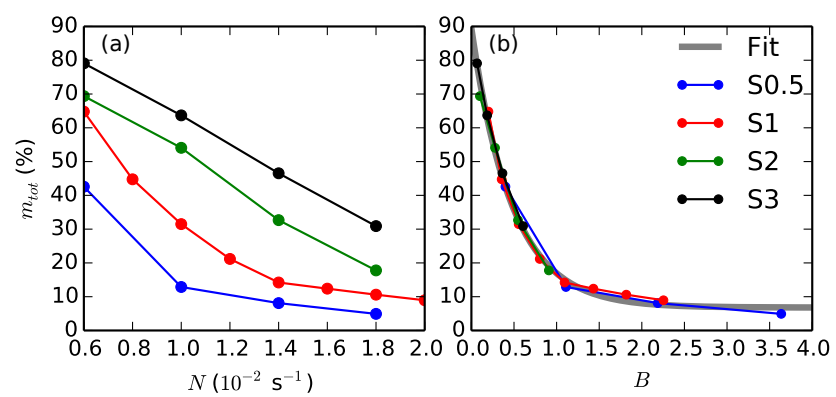

Figure 8. Total tracer mass exported out of the valley atmosphere at crest height normalized by the total tracer mass released at the surface as a function of (a) Brunt-Väisälä frequency and (b) breakup parameter for various different forcing amplitudes. A fit to the data according to Eq. (10) is shown in (b).

quired to remove the inversion, evaluated at sunrise:

$Q_{\text {req }}=L_{y} c_{\mathrm{p}} \int_{0}^{h_{\mathrm{c}}} \rho(z)\left(\Theta_{E}-\Theta(z)\right) W(z) \mathrm{d} z$,

and $Q_{\text {prov }}^{\text {pre }}$, the energy provided by the surface sensible heat flux until the time of the breakup:

$Q_{\text {prov }}^{\text {pre }}=\int_{t_{\mathrm{r}}}^{t_{\mathrm{b}}} \int_{A} H_{S}(t, x, y) \mathrm{d} x \mathrm{~d} y \mathrm{~d} t$.

Here, $H_{S}(t, x, y)$ is the sensible heat flux at the surface, $A$ is the surface area of the valley, $t_{\mathrm{r}}$ and $t_{\mathrm{b}}$ the times of sunrise and breakup respectively, $\rho$ is the air density, $L_{y}$ the length of the valley, $h_{\mathrm{c}}$ the crest height, $\Theta_{E}=\Theta\left(z=h_{\mathrm{c}}\right)$, and $W(z)$ is the width of the valley as a function of $z$.

Combining Eq. (6) and (7) and assuming $Q_{\text {req }} \sim Q_{\text {prov }}^{\text {pre }}$ (see Leukauf et al. (2015) for the rationale of this approximation) one can see that a strong forcing and a weak initial stratification lead to an early breakup. However, further conclusions for the venting potential cannot be drawn from this calculation. Moreover, not only the breakup time but also the forcing strength after the breakup is important for the total export of tracer mass since it will determine the strength of the vertical transport once the valley atmosphere is neutral.

It is therefore useful to calculate the total energy provided until sunset $t_{\mathrm{s}}$ :

$Q_{\text {prov }}=\int_{t_{\mathrm{r}}}^{t_{\mathrm{s}}} \int_{A} H_{S}(t, x, y) \mathrm{d} x \mathrm{~d} y \mathrm{~d} t$.

The ratio of required and provided energy,

$B=\frac{Q_{\text {req }}}{Q_{\text {prov }}}$,

is a non-dimensional parameter. Values of $B \gtrsim 1$ describe conditions with strong stability and/or weak forcing that 
prevent an inversion breakup. In contrast, $B \lesssim 1$ implies weak stability and/or strong forcing that enables an inversion breakup. Hence, the smaller $B$, the earlier the breakup occurs and the larger the tracer export is expected to be. For this reason we call $B$ the breakup parameter. As a first approximation one can say that for $B=1$, the valley reaches the breakup just before sunset. However, this is only a crude approximation since slope winds export energy out of the valley as well and reduce $Q_{\text {prov }}$. The exported heat leads to an increase of $\Theta_{E}$, the potential temperature above the valley, in time which causes $Q_{\text {req }}$ to increase as well. Hence, $B<1$ is typically required for the breakup when calculating $B$ according to Eq. (9) (Leukauf et al., 2015). The breakup parameter can be used to describe the combined effect of stability and forcing on the total tracer mass export out of the valley. Figure $8 \mathrm{~b}$ illustrates that this export, calculated for all simulations, is only a function of $B$. The corresponding exponential curve fitted to this data is given by

$m_{\text {tot }}=a \exp (-b B)+c$,

with $a=82.54, b=2.098$, and $c=6.79$. The export of tracer mass, which reaches values of $80 \%$ for S3N06, decreases strongly with increasing $B$ and passes the $50 \%$ mark at $B \approx 0.3$. At $B=1.0$ it has decreased to about $15 \%$. Hence, an early breakup (i.e. $B \ll 1$ ) is required for an effective venting by convection.

\section{Discussion}

In general, an initially stably stratified valley atmosphere, which is heated from the surface can be divided into three volumes: a convective boundary layer, a slope wind layer and a stable core. These layers change in time and various flow regimes can be identified (Leukauf et al., 2015). Together with the neutral layer above the valley atmosphere, these three volumes compose the boundary layer over mountainous terrain.

The definition of these volumes are based on the crest height, CBL height and slope wind layer depth $d_{\mathrm{swl}}$. The height of the vertical heat flux minimum is used to define the latter two parameters (cf. Sect. 2.3). Three different possible definitions for $d_{\text {swl }}$ have been presented in Sect. 2, and they generally lead to rather similar results (cf. Fig. 2). As expected, the largest differences occur shortly before the breakup, when the stable core is almost removed and borders between CBL and slope wind layer become diffuse. The implications of using a definition of $d_{\text {swl }}$ based on the $\theta$-gradient criterion instead of the heat-flux criterion is discussed below.

The mechanisms responsible for tracer mass transportation between the different valley volumes are up-slope winds, entrainment, HIs and recirculation (cf. Fig. 1). Most of the tracer mass emitted on the valley floor is transported into the slope wind layer during the course of the day, but whether the tracer is predominately exported at crest height or transported horizontally into the stable core and recirculates is

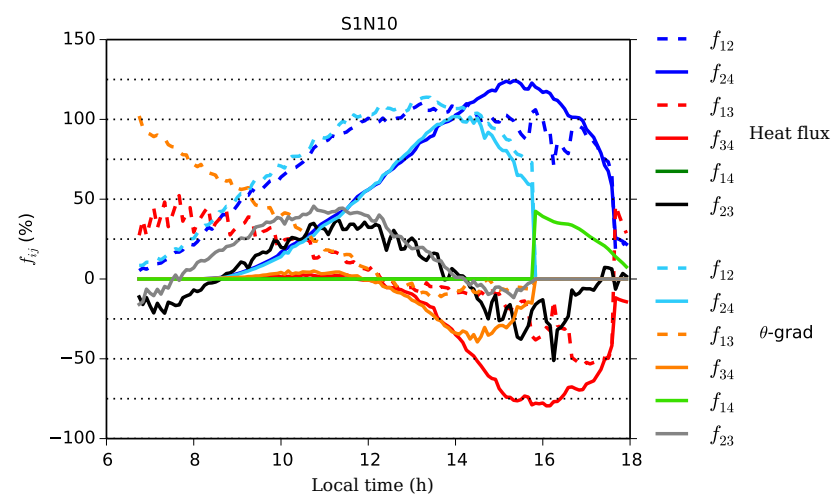

Figure 9. As in Fig. 4d, but comparing normalized bulk tracer mass fluxes for simulations $\mathrm{S} 1 \mathrm{~N} 10$ based on the heat-flux criterion (dark colours) and on the $\theta$-gradient criterion (light colours).

strongly dependent on stability and forcing amplitude (cf. Fig. 5). The HI above the boundary layer, which causes the horizontal transport into the stable core and enables a recirculation back into the CBL is a major contributor to the tracer mass flux from the slope wind layer to the stable core. For this reason, the magnitudes of the associated fluxes depend to some extent on the definition of boundary layer height and $d_{\text {swl }}$. Comparing results shown in Fig. 4 with fluxes calculated using CBL heights and slope wind layer depths according to the $\theta$-gradient criterion shows that the relative differences between the two calculation methods are generally relatively small, with some exceptions as illustrated in Fig. 9 for the simulation S1N10. If the $\theta$-gradient criterion is used, the normalized vertical flux in the middle of the valley $\left(f_{13}\right)$ is up to 3 times larger in the first $2 \mathrm{~h}$ of simulation. This is due to a much lower initial CBL height using this criterion. Flux $f_{23}$ is also larger in the early morning hours but the differences decrease and reach zero at noon. The vertical fluxes in and out of the slope wind layer $\left(f_{12}\right.$ and $\left.f_{24}\right)$ are almost identical for both criteria. The largest difference between the two definitions is the fact that the breakup for S1N10 is reached at 16:00 LT when using the $\theta$-gradient criterion, but not reached when using the heat-flux criterion. Depending on the criterion, volumes representing the slope wind layer and the stable core have a different width a few hours before the breakup, which affects the fluxes representing the export and re-import of tracer mass. Fluxes according to the $w$ criterion have not been calculated since the slope wind layer depth is similar to that of the heat-flux criterion. Overall, the sensitivity of the fluxes to various slope wind layer/CBL criteria is smaller than the sensitivity to stability and forcing. Also, the total export of tracer mass at crest height is independent of these definitions.

The horizontal and vertical transport of tracer has been studied for a wide range of stability conditions and forcing amplitudes, but only for a very idealized framework. Soundings with a constant stability throughout the atmosphere are 
used to initialize the model, which is not very realistic. Multiple elevated inversions are common (Emeis et al., 2007; Rotach et al., 2015), and may cause secondary HIs, which would contribute to $f_{23}$ and lead to more tracer being intruded into the stable core at higher levels. Furthermore, we have not considered the effect of a residual layer. Often, the valley atmosphere in the morning consists of a cold pool close to the ground, a residual layer around crest height and a stable layer aloft (De Wekker et al., 2005; Chow et al., 2006). Leukauf et al. (2015) allowed a valley atmosphere with a constant stratification to develop over $36 \mathrm{~h}$ and found a similar vertical structure at sunrise of the second day. The slope winds that develop in such a scenario are stronger above the cold pool and a large fraction of pollutants released at the valley floor are trapped until the cold pool is weakened enough. As the cold pool is eroded, we expect to see a gradual increase of the export of tracer mass and a strong increase as the breakup is reached. Such a behaviour has been mentioned by Leukauf et al. (2015), but not studied in detail. The development of HIs and, hence, the strength of $f_{23}$ is also depends on the terrain geometry. Wagner et al. (2015a) found secondary circulations within the valley (i.e. HIs) for steep and narrow valleys, but none for shallow valley geometries.

The chosen quasi-two-dimensional valley geometry, which neither allows for the development of along-valley winds nor for a plain-to-mountain circulation, is a major simplification. The mountain-to-plain circulation provides a mechanism to remove tracer from the vicinity of the mountain peaks (Wagner et al., 2015a) and it is conceivable that this 3-D transport reduces the reimport of pollutants into the valley by compensating subsidence compared to a 2-D case. A corresponding weaker downward transport of tracer into the valley in a 3-D simulation compared to a 2-D one was noticed by Lehner and Gohm (2010). However, the alongvalley wind also enables transport of either clean or polluted air from the adjacent plain into the valley. Hence, the net effect of a 3-D valley geometry on the pollution inside a valley strongly depends on the amount of pollutants over the adjacent plain.

Due to the symmetry of the problem considered in this study, the two-slope wind layers have been treated as a single volume. In reality, asymmetric flow structures have been reported, e.g. in case of asymmetric solar forcing (Harnisch and Gohm, 2009; Gohm et al., 2009) and valley curvature (Weigel et al., 2007). Along-slope variations of the slope wind layer due to changes in slope angle and soil properties are also neglected, although strong gradients in these properties could also cause HIs (Lehner and Gohm, 2010). However, for the total export of tracer mass, the detailed structure within the valley is less important. It is likely that vertical fluxes associated with two asymmetric slope wind layers will on average export as much pollutants as those of two symmetric layers. Further, a spatially constant forcing will on average cause a similar result as a complex, variable forcing as long as the bulk heat input into the valley atmosphere is comparable (Rotach et al., 2015). In other words, the exchange of properties between the valley and the atmosphere above the valley is mainly determined by bulk properties subsumed in the $B$ parameter rather than by spatial and temporal variability of stratification and surface heating. Hence, we believe that the highly idealized approach chosen in this study is suitable for drawing more general conclusions.

We have shown that the export of tracer out of the valley is well described by our breakup parameter $B=Q_{\text {req }} / Q_{\text {prov }}$ (Fig. 8b). Princevac and Fernando (2008) introduced a similar parameter, $B_{\mathrm{PF}}=\frac{N^{3} H^{2}}{q_{0}}$, that essentially describes the ratio between the static stability of the valley atmosphere and the surface sensible heat flux ${ }^{1}$. Here we recall that the stability is proportional to $Q_{\text {req }}$ and the surface sensible heat flux proportional to $Q_{\text {prov }}$ (Eq. 8). Based on laboratory experiments, Princevac and Fernando (2008) concluded that the destruction of the cold-air pool in a valley is determined by convection for low values of $B_{\mathrm{PF}}$ and by HIs for high values of $B_{\mathrm{PF}}$. Similarly, we found that HIs occur predominantly for large values of $B$. However, this is also the situation when a complete breakup of the valley inversion does not occur. It appears that HIs alone are not efficient enough to neutralize the stratification in a valley.

It is known that thermally driven winds can provide an effective venting mechanism under favourable conditions. The number of turnovers of valley air mass during daytime lies between zero and five, depending on the forcing amplitude (see Rotach et al. (2015) for an overview). We have shown that stratification is a crucial parameter to determine the efficiency of vertical exchange processes in addition to the forcing amplitude. This is in good agreement with the work of Segal et al. (1988), Whiteman and McKee (1982), and Chemel et al. (2016). The breakup parameter $B$ is suitable to describe the mutual effects of stability and forcing for the vertical export of tracer mass on a daily basis (cf. Sect. 3.5). It is important to note that the export of tracer mass decreases exponentially with increasing $B$ since this parameter determines the strength of the slope winds. However, we need to emphasize that the number of turnovers of valley air mass is not a measure of the venting efficiency since tracer mass may be reimported into the valley from above. Consequently, the exchange efficiency as discussed so far (given turnover numbers of up to five) gives an overly optimistic picture of the actual exchange of tracer mass. However, using an atmospheric sounding at sunrise, knowledge on the valley geometry and an estimation of the average surface sensible heat flux, one can compute the breakup parameter $B$ (Eq. 9) and consequently the fraction of the emitted pollution that is exported out of the valley atmosphere during daytime.

An interesting question is whether the presented results for a passive tracer can be applied to transport processes of heat and moisture as well. In principle, both heat and moisture are

\footnotetext{
${ }^{1}$ More specifically, $N$ is the Brunt-Väisälä frequency, $H$ is the inversion height, and $q_{0}$ the buoyancy flux at the surface.
} 
transported by the slope flows; hence, it is reasonable to assume similarities. However, heat is released over the whole terrain surface area in contrast to the passive tracer, which is released in our case only at the valley floor. An earlier onset of the export of heat has therefore to be expected. At the same time, the exported heat leads to an increase of the temperature above the valley, which affects the breakup of the valley inversion (Leukauf et al., 2015) and therefore the export of heat. The transport of moisture is even more complicated since moisture is no passive tracer. Clouds form as soon as water vapour condensates, affecting the short-wave radiative flux at the surface and therefore the sensible heat flux. Nevertheless, it seems reasonable to expect that the moisture transport is qualitatively similar to the transport of a passive tracer as long as no condensation occurs.

\section{Conclusions}

The evolution of a daytime valley boundary layer and the transport of a passive tracer between three different valley sub-volumes (convective boundary layer, slope wind layer, stable core) and the atmosphere above the valley is investigated by means of large eddy simulations. The model is initialized with a constant Brunt-Väisälä frequency and the surface sensible heat flux is prescribed by a sine-shaped function. Numerous simulations have been performed for initial Brunt-Väisälä frequencies $N$ ranging between $N=0.006$ and $0.02 \mathrm{~s}^{-1}$ and for surface heat flux amplitudes between 62.5 and $375 \mathrm{~W} \mathrm{~m}^{-2}$. These are the main results:

- A horizontal intrusion forms above the convective boundary layer at the transition zone between the slope wind layer and the stable core. This phenomenon is in agreement with the circulation described by Vergeiner's idealized slope-flow model (Vergeiner, 1982; Vergeiner and Dreiseitl, 1987), which predicts horizontal air mass export from the slope wind layer as a result of vertically increasing ambient stability between the convective boundary layer and the stable core. By this circulation, tracer mass released at the valley floor is intruded into the stable core.

- For a given heat flux amplitude, the efficiency of the vertical tracer mass transport strongly depends on the static stability. For example, for a typical forcing amplitude of $125 \mathrm{~W} \mathrm{~m}^{-2}$, the net export of tracer mass out of the valley atmosphere during the course of the day may range between 10 and $65 \%$ depending on whether the atmospheric stability is considerably higher or lower than the value of the standard atmosphere $\left(N=0.01 \mathrm{~s}^{-1}\right)$ respectively.

- The vertical transport of tracer mass from the convective boundary layer into the slope wind layer primarily depends on the forcing amplitude. A stronger forcing leads to an earlier onset of the associated tracer mass flux and a sharper increase in time. The horizontal flux from the slope wind layer into the stable core and the export at crest height depend on both forcing amplitude and initial stability. The export decreases drastically with increasing stability and decreasing forcing while the horizontal transport increases.

- There is a similarity between simulations with different stability conditions and forcing amplitudes in the sense that a combination of weak forcing and weak stability leads roughly to the same export of tracer mass during the course of the day as a combination of strong forcing and strong stability.

- A so-called breakup parameter $B$ has been introduced that combines the effect of stability and forcing on the total export of tracer mass. It is defined as the ratio of energy required to neutralize the stratification in the valley to the energy provided by the surface sensible heat flux over the course of the day.

- An early breakup of the valley inversion is required for effective venting of pollutants. Half of the tracer mass released at the surface is exported for $B \approx 0.3$, whereas only about $15 \%$ is exported for $B=1$.

Although this study is limited by the choice of idealized assumptions, i.e. quasi-two-dimensional topography, initially a vertically constant stratification and horizontally homogeneous surface heating, it provides an overview of the magnitudes of pollutant transport for a wide variety of stability conditions and forcing amplitudes. Also, we propose a single parameter, which describes the total export of tracer mass over the course of the day. In future studies, it would be desirable to test the dependence of pollutant venting on the breakup parameter for different valley topographies, including 3-D geometries, and to clarify whether the export of other quantities, such as heat and moisture, could be described by a similar relation. The impact of more realistic profiles of atmospheric stability on horizontal and vertical fluxes would be another important topic for a future study.

\section{Data availability}

All data that are required to reproduce the numerical simulations presented in this article can be found in the Supplement. This includes changes to the WRF model code, namelist files, and input soundings as well as compiler information.

The Supplement related to this article is available online
at doi:10.5194/acp-16-13049-2016-supplement. 
Author contributions. D. Leukauf designed the numerical experiments, carried them out, and prepared the manuscript. A. Gohm and M. W. Rotach provided suggestions for the design of the experiments, recommended relevant literature, discussed the results with the main author, and contributed to the manuscript by critical comments on text and figures, and proof reading.

Acknowledgements. This work was supported by the Austrian Science Fund (FWF) under grant P23918-N21, by the Austrian Federal Ministry of Science, Research and Economy (BMWFW) as part of the UniInfrastrukturprogramm of the Research Focal Point Scientific Computing at the University of Innsbruck, and by a PhD scholarship of the University of Innsbruck in the framework of the Nachwuchsförderung 2015. Computational results presented have been achieved in part using the Vienna Scientific Cluster (VSC). We thank two anonymous reviewers for their helpful comments.

Edited by: B. Vogel

Reviewed by: two anonymous referees

\section{References}

Catalano, F. and Cenedese, A.: High-Resolution Numerical Modeling of Thermally Driven Slope Winds in a Valley with Strong Capping, J. Appl. Meteorol. Clim., 49, 1859-1880, doi:10.1175/2010JAMC2385.1, 2010.

Catalano, F. and Moeng, C.-H.: Large-Eddy Simulation of the Daytime Boundary Layer in an Idealized Valley Using the Weather Research and Forecasting Numerical Model, Bound-Lay. Meteorol., 137, 49-75, doi:10.1007/s10546-010-9518-8, 2010.

Chazette, P., Couvert, P., Randriamiarisoa, H., Sanak, J., Bonsang, B., Moral, P., Berthier, S., Salanave, S., and Toussaint, F.: Three-dimensional survey of pollution during winter in French Alps valleys, Atmos. Environ., 39, 1035-1047, doi:10.1016/j.atmosenv.2004.10.014, 2005.

Chemel, C., Arduini, G., Staquet, C., Largeron, Y., Legain, D., Tzanos, D., and Paci, A.: Valley heat deficit as a bulk measure of wintertime particulate air pollution in the Arve River Valley, Atmos. Environ., 128, 208-215, doi:10.1016/j.atmosenv.2015.12.058, 2016.

Chow, F. K., Weigel, A. P., Street, R. L., Rotach, M. W., and Xue, M.: High-Resolution Large-Eddy Simulations of Flow in a Steep Alpine Valley. Part I: Methodology, Verification, and Sensitivity Experiments, J. Appl. Meteorol. Clim., 45, 63-86, doi:10.1175/JAM2322.1, 2006.

de Franceschi, M. and Zardi, D.: Study of wintertime high pollution episodes during the Brenner-South ALPNAP measurement campaign, Meteorol. Atmos. Phys., 103, 237-250, doi:10.1007/s00703-008-0327-2, 2009.

De Wekker, S. F. J. and Kossmann, M.: Convective Boundary Layer Heights Over Mountainous Terrain - A Review of Concepts, Front. Earth Sci., 3, doi:10.3389/feart.2015.00077, 2015.

De Wekker, S. F. J., Steyn, D. G., Fast, J. D., Rotach, M. W., and Zhong, S.: The performance of RAMS in representing the convective boundary layer structure in a very steep valley, Environ. Fluid Mech., 5, 35-62, doi:10.1007/s10652-005-8396-y, 2005.
Deardorff, J. W.: Stratocumulus-capped mixed layers derived from a three-dimensional model, Bound-Lay. Meteorol., 18, 495-527, doi:10.1007/BF00119502, 1980.

Emeis, S., Jahn, C., Münkel, C., Münsterer, C., and Schäfer, K.: Multiple atmospheric layering and mixing-layer height in he Inn valley observed by remote sensing, Meteorol. Z., 16, 415-424, doi:10.1127/0941-2948/2007/0203, 2007.

Fast, J. D. and Zhong, S.: Meteorological factors associated with inhomogeneous ozone concentrations within the Mexico City basin, J. Geophys. Res.-Atmos., 103, 18927-18946, doi:10.1029/98JD01725, 1998.

Gohm, A., Harnisch, F., Vergeiner, J., Obleitner, F., Schnitzhofer, R., Hansel, A., Fix, A., Neininger, B., Emeis, S., and Schäfer, K.: Air Pollution Transport in an Alpine Valley: Results From Airborne and Ground-Based Observations, Bound-Lay Meteorol., 131, 441-463, doi:10.1007/s10546-009-9371-9, 2009.

Harnisch, F. and Gohm, A.: Spatial distribution of aerosols in the Inn Valley atmosphere during wintertime, Meteorol. Atmos. Phys., 103, 223-235, doi:10.1007/s00703-008-0318-3, 2009.

Henne, S., Furger, M., Nyeki, S., Steinbacher, M., Neininger, B., de Wekker, S. F. J., Dommen, J., Spichtinger, N., Stohl, A., and Prévôt, A. S. H.: Quantification of topographic venting of boundary layer air to the free troposphere, Atmos. Chem. Phys., 4, $497-$ 509, doi:10.5194/acp-4-497-2004, 2004.

Kossman, M., Corsmeier, U., De Wekker, S. F. J., Fiedler, F., Vögtlin, R., Kalthoff, N., Günsten, H., and Neininger, B.: Observations of handover processes between the atmospheric boundary layer and the free troposphere over mountainous terrain, Contr. Atmos. Phys., 72, 329-350, 1999.

Lang, M. N., Gohm, A., and Wagner, J. S.: The impact of embedded valleys on daytime pollution transport over a mountain range, Atmos. Chem. Phys., 15, 11981-11998, doi:10.5194/acp15-11981-2015, 2015.

Lehner, M. and Gohm, A.: Idealised Simulations of Daytime Pollution Transport in a Steep Valley and its Sensitivity to Thermal Stratification and Surface Albedo, Bound-Lay. Meteorol., 134, 327-351, doi:10.1007/s10546-009-9442-y, 2010.

Leukauf, D., Gohm, A., Rotach, M. W., and Wagner, J. S.: The Impact of the Temperature Inversion Breakup on the Exchange of Heat and Mass in an Idealized Valley: Sensitivity to the Radiative Forcing, J. Appl. Meteorol. Clim., 54, 2199-2216, doi:10.1175/JAMC-D-15-0091.1, 2015.

Lu, R. and Turco, R. P.: Air Pollutant Transport in a Coastal Environment, Part I: Two-Dimensional Simulations of SeaBreeze and Mountain Effects, J. Atmos. Sci., 51, 2285-2308, doi:10.1175/1520-0469(1994)051<2285:APTIAC>2.0.CO;2, 1994.

Lu, R. and Turco, R. P.: Air pollutant transport in a coastal environment-II. Three-dimensional simulations over Los Angeles basin, Atmos. Environ., 29, 1499-1518, doi:10.1016/13522310(95)00015-Q, 1995.

Monin, A. S. and Obukhov, A. M.: Basic laws of turbulent mixing in the ground layer of the atmosphere, Akad. Nauk SSSR Geofiz. Inst. Tr., 151, 163-187, 1954.

Princevac, M. and Fernando, H. J. S.: Morning breakup of cold pools in complex terrain, J. Fluid Mech., 616, 99-109, doi:10.1017/S0022112008004199, 2008.

Rendón, A. M., Salazar, J. F., Palacio, C. A., and Wirth, V.: Temperature Inversion Breakup with Impacts on Air Quality in Urban 
Valleys Influenced by Topographic Shading, J. Appl. Meteorol. Clim., 54, 302-321, doi:10.1175/JAMC-D-14-0111.1, 2014.

Reuten, C., Steyn, D. G., and Allen, S. E.: Water tank studies of atmospheric boundary layer structure and air pollution transport in upslope flow systems, J. Geophys. Res.-Atmos., 112, D11114, doi:10.1029/2006JD008045, 2007.

Rotach, M. W. and Zardi, D.: On the boundary-layer structure over highly complex terrain: Key findings from MAP, Q. J. Roy. Meteor. Soc., 133, 937-948, doi:10.1002/qj.71, 2007.

Rotach, M. W., Andretta, M., Calanca, P., Weigel, A. P., and Weiss, A.: Boundary layer characteristics and turbulent exchange mechanisms in highly complex terrain, Acta Geophys., 56, 194-219, doi:10.2478/s11600-007-0043-1, 2007.

Rotach, M. W., Gohm, A., Lang, M. N., Leukauf, D., Stiperski, I., and Wagner, J. S.: On the Vertical Exchange of Heat, Mass, and Momentum Over Complex, Mountainous Terrain, Front. Earth Sci., 3, doi:10.3389/feart.2015.00076, 2015.

Schmidli, J.: Daytime Heat Transfer Processes over Mountainous Terrain, J. Atmos. Sci., 70, 4041-4066, doi:10.1175/JAS-D-13083.1, 2013.

Schmidli, J. and Rotunno, R.: Mechanisms of Along-Valley Winds and Heat Exchange over Mountainous Terrain, J. Atmos. Sci., 67, 3033-3047, doi:10.1175/2010JAS3473.1, 2010.

Schnitzhofer, R., Norman, M., Wisthaler, A., Vergeiner, J., Harnisch, F., Gohm, A., Obleitner, F., Fix, A., Neininger, B., and Hansel, A.: A multimethodological approach to study the spatial distribution of air pollution in an Alpine valley during wintertime, Atmos. Chem. Phys., 9, 3385-3396, doi:10.5194/acp-93385-2009, 2009.

Segal, M., Yu, C.-H., Arritt, R., and Pielke, R.: On the impact of valley/ridge thermally induced circulations on regional pollutant transport, Atmos. Environ., 22, 471-486, doi:10.1016/00046981(88)90193-X, 1988.

Seibert, P., Beyrich, F., Gryning, S.-E., Joffre, S., Rasmussen, A., and Tercier, P.: Review and intercomparison of operational methods for the determination of the mixing height, Atmos. Environ., 34, 1001-1027, doi:10.1016/S1352-2310(99)00349-0, 2000.

Serafin, S. and Zardi, D.: Structure of the Atmospheric Boundary Layer in the Vicinity of a Developing Upslope Flow System: A Numerical Model Study, J. Atmos. Sci., 67, 1171-1185, doi:10.1175/2009JAS3231.1, 2010.
Silcox, G. D., Kelly, K. E., Crosman, E. T., Whiteman, C. D., and Allen, B. L.: Wintertime PM2.5 concentrations during persistent, multi-day cold-air pools in a mountain valley, Atmos. Environ., 46, 17-24, doi:10.1016/j.atmosenv.2011.10.041, 2012.

Sullivan, P. P., Moeng, C.-H., Stevens, B., Lenschow, D. H., and Mayor, S. D.: Structure of the Entrainment Zone Capping the Convective Atmospheric Boundary Layer, J. Atmos. Sci., 55, 3042-3064, doi:10.1175/15200469(1998)055<3042:SOTEZC>2.0.CO;2, 1998.

Suppan, P., Schäfer, K., Vergeiner, J., Emeis, S., Obleitner, F., and Griesser, E.: Assessment of air pollution in the vicinity of major alpine routes, in: Highway and Urban Environment, edited by: Kauffman, J. M., Morrison, G. M., and Rauch, S., Springer Netherlands, Dordrecht, the Netherlands, 12, 203-214, doi:10.1007/978-1-4020-6010-6_19, 2007.

Vergeiner, D. I. and Dreiseitl, D. E.: Valley winds and slope winds Observations and elementary thoughts, Meteorol. Atmos. Phys., 36, 264-286, doi:10.1007/BF01045154, 1987.

Vergeiner, I.: Eine energetische Theorie der Hangwinde, 17. Int. Tag. Alpine Meteorol., 189-191, 1982.

Wagner, J. S., Gohm, A., and Rotach, M. W.: The Impact of Horizontal Model Grid Resolution on the Boundary Layer Structure over an Idealized Valley, Mon. Weather Rev., 142, 3446-3465, doi:10.1175/MWR-D-14-00002.1, 2014.

Wagner, J. S., Gohm, A., and Rotach, M. W.: The impact of valley geometry on daytime thermally driven flows and vertical transport processes, Q. J. Roy. Meteor. Soc., 141, 1780-1794, doi:10.1002/qj.2481, 2015a.

Wagner, J. S., Gohm, A., and Rotach, M. W.: Influence of alongvalley terrain heterogeneity on exchange processes over idealized valleys, Atmos. Chem. Phys., 15, 6589-6603, doi:10.5194/acp15-6589-2015, $2015 \mathrm{~b}$.

Weigel, A. P., Chow, F. K., and Rotach, M. W.: The effect of mountainous topography on moisture exchange between the "surface" and the free atmosphere, Bound.-Lay. Meteorol., 125, 227-244, doi:10.1007/s10546-006-9120-2, 2007.

Whiteman, C. D. and McKee, T. B.: Breakup of Temperature Inversions in Deep Mountain Valleys: Part II, Thermodynamic Model, J. Appl. Meteorol., 21, 290-302, doi:10.1175/15200450(1982)021<0290:BOTIID>2.0.CO;2, 1982. 